\title{
In planta dynamics, transport-biases and endogenous functions of mobile siRNAs in Arabidopsis
}

Christopher A. Brosnan ${ }^{1, \# \dagger}$, Emanuel A. Devers ${ }^{1, \#, \neq, I D}$, Alexis Sarazin ${ }^{1}$, Peiqi Lim ${ }^{1, *}$, Satu Lehesranta ${ }^{2}$, Yrjö Helariutta ${ }^{2,3, I D}$, Olivier Voinnet ${ }^{1, \mathrm{ID},{ }^{*}}$

${ }^{1}$ Department of Biology, Swiss Federal Institute of Technology (ETH), Universitätsstrasse 2, 8092 Zürich, Switzerland.

${ }^{2}$ Institute of Biotechnology, University of Helsinki, PO Box 65, Helsinki, FIN-00014, Finland.

${ }^{3}$ Sainsbury Laboratory, University of Cambridge, Bateman Street, Cambridge, CB2 1LR, United Kingdom.

†present address: Queensland Alliance for Agriculture and Food Innovation, The University of Queensland, Brisbane, 4072, Australia

Fpresent address: Department of Environmental Sciences, Swiss Federal Institute of Technology (ETH), Schmelzbergstrasse 9, 8092 Zürich, Switzerland.

Fpresent address: QIAGEN Singapore Pte. Ltd. 8 Commonwealth Lane \#02-02 Singapore 149555 Singapore

ID CAB: https://orcid.org/0000-0002-6589-985X EAD: https://orcid.org/0000-00016237-8867; YH: https://orcid.org/0000-0002-7287-8459, OV: https://orcid.org/0000$\underline{0001-6982-9544}$

"These authors contributed equally

*Correspondence to: Olivier Voinnet (voinneto@ethz.ch) 


\section{ABSTRACT}

-In RNA interference (RNAi), small-interfering (si)RNAs processed from doublestranded RNA guide ARGONAUTE(AGO) proteins to silence sequencecomplementary RNA/DNA. Plant RNAi can propagate locally and systemically, but despite recent mechanistic advances, basic questions/hurdles remain unaddressed. For instance, RNAi is inferred to diffuse through plasmodesmata, yet how its dynamics in planta compares with that of established symplastic-diffusion markers remains unknown. Also unknown is why select siRNA species, or size-classes thereof, are recovered in RNAi-recipient tissues, yet only under some experimental settings. Finally, RNAi shootward movement in micro-grafted Arabidopsis necessary to study its presumptive transgenerational effects- has not been achieved thus far and endogenous functions of mobile RNAi remain scarcely documented.

-Focusing on non-amplified RNAi in Arabidopsis, we show here that $(i)$ transgenic RNAi-movement, although symplasmic, only partially recapitulates the diffusion pattern of free GFP in planta, (ii) the presence/absence of specific AGOs in incipient/traversed/recipient tissues likely explains the apparent siRNA-selectivity observed during vascular movement, (iii) stress application allows endo-siRNA translocation against the shoot-to-root phloem flow, and (iv) mobile endo-siRNAs generated from a single inverted-repeat $(I R)$ locus, have the potential to regulate hundreds of transcripts.

-Our results close important knowledge-gaps, rationalize previously-noted inconsistencies between mobile RNAi settings, and provide a framework for functional endo-siRNA studies. 


\section{INTRODUCTION}

During RNA interference (RNAi), the RNase-III Dicer processes cohorts of 20-24-nt siRNAs from long double-stranded (ds)RNA. Loaded into AGOs, siRNAs guide endonucleolysis ('slicing') and/or translational repression of sequencecomplementary mRNAs. Single-species of endogenous micro(mi)RNAs also are produced by Dicer from genome-encoded imperfect stem-loop precursors (Bologna and Voinnet, 2014). The present study focuses on siRNA-directed RNAi, which can also occur endogenously via "endo-siRNAs". In plants, endo-siRNAs derive from IR loci, converging transcription or RNA-dependent-RNA-polymerase(RDR) activities (Bologna and Voinnet, 2014). The Arabidopsis endo-siRNA's bulk comprises 24-nt species processed from transposable element(TE)- and repeat-derived-dsRNA by DCL3 (Xie et al., 2004), one of four Dicer-like proteins (DCL1-4). Loaded into AGO4clade (AGO4/6/9) effectors, these guide RNA-directed-DNA-methylation(RdDM) in all cytosine contexts $(\mathrm{CHH}, \mathrm{CHG}, \mathrm{CG}$; $\mathrm{H}$ : any nucleotide but $\mathrm{G})$, causing transcriptional-gene-silencing (TGS) predominantly at their loci-of-origin (Law and Jacobsen, 2010). DCL4 and DCL2 produce respectively 21-nt and 22-nt endosiRNAs from a much smaller set of IRs and RDR6-amplified TRANS-ACTING-(ta) or PHASED-(pha)siRNA loci(TAS and PHAS) (Gasciolli et al., 2005; Henderson et al., 2006; Liu et al., 2020). Both also convert viral dsRNA into antiviral, virus-derived (v)siRNAs (Bouché et al., 2006; Deleris et al., 2006). Like miRNAs, tasi/phasi/vsiRNAs are mainly loaded into AGO1/AGO2 to execute posttranscriptional-gene-silencing (PTGS).

In plants and some metazoans, RNAi can act non-cell-autonomously, as first recognized in tobacco displaying dynamic transgene-silencing evoking the photo- 
assimilates' source-to-sink allocation path (Palauqui et al., 1996; Voinnet and Baulcombe, 1997). Graft-transmission of silencing from rootstocks to scions (Palauqui et al., 1997) was later recapitulated in micro-grafted Arabidopsis (Brosnan et al., 2007). Seldom examples of non-transgenic i.e. endo-RNAi movement include TAS3 tasiRNAs during leaf polarization (Chitwood et al., 2009; Schwab et al., 2009), siRNAs derived from TEs or endo-IRs (Devers et al., 2020; Molnar et al., 2010) and phasiRNAs through inter-species grafts (Li et al., 2021). While grafting provides a simple readout of long-distance RNAi transport, its implementation and interpretations have limitations. As a major physiological stress, grafting likely exaggerates inter-organ photoassimilates' fluxes (Kehr, 2013), which questions the significance of sRNA-target gene regulations in graft-recipient tissues ( $\mathrm{Li}$ et al., 2021). Micro-grafting-inherent caveats also likely explain why endo-RNAi is grafttransmitted from shoot-to-root, not root-to-shoot, in Arabidopsis. In micro-grafts, rootstocks are made of non-photosynthetic hypocotyls and roots, whereas scions produce photosynthetic leaves. The ensuing source(scions)-to-sink(rootstocks) photoassimilates' flux likely prevents optimal shootward RNAi-transport. The more challenging method of stem-grafting using adult plants allows shootward transport of siRNA-based phenotypes (Kundariya et al., 2020). While it imparts systemic DNA/RNA-level regulations (Lewsey et al., 2016; Molnar et al., 2010), micro-graftingmediated shoot-to-root transmission in Arabidopsis conflicts with the popular notion that mobile endo-RNAi endows heritable epigenetic modifications; indeed, root apices, unlike shoot apices, do not produce a germline or embryos (Conine and Rando, 2021). Shootward movement via reproductive stem-grafting enables, by contrast, transgenerational inheritance in Arabidopsis, although a direct role for mobile RNAi was not strictly demonstrated (Kundariya et al., 2020). Enhancing endo- 
siRNA production in micro-grafted rootstocks using, e.g. stress, could revalorize micro-grafting for such studies by possibly enabling root-to-shoot RNAi transport against the suboptimal photoassimilates' flux.

Heterografting between plant species/ecotypes coupled to sRNA deepsequencing and single-nucleotide-polymorphism mapping helps discerning which, among rootstock's and scion's sRNAs, endow mobile RNAi (Li et al., 2021; Molnar et al., 2010). Importantly, this cannot discriminate siRNAs derived from highly conserved, repeated TEs. A common solution in Arabidopsis is to use homo-grafted dcl234 triple-mutant rootstocks devoid of endo-siRNAs. Curiously, siRNA species from only some loci are retrieved in dcl234 recipient roots (Molnar et al., 2010), suggesting that selectivity might accompany vascular RNAi movement (Maizel et al., 2020). However, the dcl234 background itself might introduce this bias, an equally plausible, albeit untested possibility. An apparent bias also exists regarding which siRNA species, among the global products of DCL2,-3,-4 in silencing-emitting tissues, correlates with long-distance RNAi. Supporting early work on systemic transgene silencing (Hamilton et al., 2002), Molnar et al., reported that 24-nt siRNAs predominate in RNAi-recipient rootstocks of micro-grafted Arabidopsis. This bias was not observed, however, in a recent study of three distinct mobile RNAi sources (discussed subsequently), including via micro-grafting (Devers et al., 2020), underscoring discrepancies still unexplained to date.

In attempting to address the long-debated RNAi signal's identity, we recently showed that AGO-free siRNAs are necessary and sufficient for cell-to-cell and longdistance RNAi movement from an $I R$ transgene, an endo- $I R$ locus or a naturally phloem-restricted virus (Devers et al., 2020). Moreover, deep-sequencing analyses in RNAi-incipient versus RNAi-recipient tissues showed that a fraction (transgene, 
virus) or majority (endo-IR) of mobile siRNAs is selectively "consumed" across traversed cells by being irreversibly loaded into cell-autonomous AGOs. A tight correlation was indeed established between siRNA cell-to-cell mobility and the 5'-nt identity of individual siRNAs, which, together with their length, largely dictates sRNA:AGO-loading specificities. Hence, 5'U/5'A-terminal siRNAs were substantially more consumed (i.e. less mobile) than $5^{\prime} \mathrm{C} / 5^{\prime} \mathrm{G}$-terminal siRNAs, respectively reflecting expression of $A G O 1$ and $A G O 2 / A G O 4$, but not of AGO5 or any known 5'Gspecific AGO, in leaves (Devers et al., 2020). While its impact on vasculature-based long-distance transport remains unexplored, consumption is best accommodated in a situation where siRNAs should move via the symplasm, the cytosolic continuum shared, via plasmodesmata (PDs), by most plant cells. Indirectly supporting this notion, naturally symplasmically-isolated stomata guard-cells resist mobile RNAi (Himber et al., 2003; Voinnet et al., 1998). Moreover, mutations increasing PDs' aperture enhance mobile transgenic RNAi (Kobayashi and Zambryski, 2007) which is decreased/increased in adult Arabidopsis by knockout/overexpression of receptorlike-kinases of which a subfraction is PD-associated (Rosas-Diaz et al., 2018). Nonetheless, proof that RNAi spreads symplasmically awaits the physical obstruction of PDs via spatio/temporally-controlled callose deposition, which indeed impedes endodermis-to-stele mobility of miR165a or its precursor(s) in Arabidopsis root tips (Vatén et al., 2011). Whether this result can be extrapolated to other miRNAs or indeed to the siRNA populations studied here remains especially unclear.

RNAi movement is often divided into distinct cell-to-cell and long-distance (i.e. vascular) processes, which, in reality, are likely intimately linked because the PDbased symplasmic continuum connects not only adjacent non-vascular cells but also the enucleated phloem-sieve-elements (SEs). This was elegantly confirmed in 
seminal studies of macromolecular transport in intact Arabidopsis involving freely diffusible(free-)GFP expressed from the phloem-companion-cell(CC)-specific promoter, pSUC2 (Imlau et al., 1999; Stadler et al., 2005). Free-GFP (1) translocates via PDs from CCs-to-SEs in photosynthetically mature i.e. phloem- $\underline{\text { Source }}$ Leave $\underline{\mathbf{s}}$ (SoLs) in which pSUC2 is active, (2) moves over long-distances within SEs along the transport phloem and (3) passively unloads and diffuses, via PDs, in photosynthetically immature i.e. phloem-Sink Leaves (SiLs) in which pSUC2 is barely active. A mobile, artificial miRNA showed distinctive differences with free-GFP in aspects of its spread (Skopelitis et al., 2018); how RNAi movement compares in planta with that of free-GFP remains unexplored, however.

Last but not least, the functional/biological significance of mobile endo-RNAi remains cryptic. Cell-to-cell spread of TAS3 tasiRNAs is thought to create gene expression gradients (Chitwood et al., 2009; Schwab et al., 2009). However, this may merely reduce transcriptional repression costs over multiple cells, or regulate processes themselves non-autonomous. Likewise, while shoot-to-root transmission of endo-siRNA-triggered RNAi is well-established in micro-grafted Arabidopsis, the biological outcome of the movement of endo-siRNAs spawned from such loci is unknown. This question is particularly relevant in intact, i.e. non-grafted, plants given the grafting-associated caveats discussed here. In this study, we endeavoured to address most of the uncertainties remaining on mobile RNAi as laid out in this introduction in order to also close important knowledge-gaps on the process. 


\section{RESULTS}

\section{siRNA movement in whole plants only partially recapitulates that of free-GFP}

We used pSUC2::tmGFP9 and pSUC2::GFP (Stadler et al., 2005), reporting spatial patterns of respectively trans-membranous-(tm, i.e. cell-autonomous)GFP and freeGFP as a non-selective marker of passive macromolecular trafficking. Mobile siRNA activity was monitored side-by-side in the pSUC2::SUL-IR RNAi line (SS; Himber et al., 2003; Devers et al. 2020). In SS, PTGS of MAGNESIUM CHELATASE(SUL) causes expanded photobleaching beyond the pSUC2 CC-specific activity domain. This reflects movement of non-amplified, AGO-free SUL-siRNAs (Devers et al., 2020) of which the DCL4-dependent 21-nt, species are necessary and sufficient for PTGS (Dunoyer et al., 2005). As reported, the tmGFP9 signal was CC-restricted in SoLs and below detection in SiLs where pSUC2 activity is low; free-GFP, however, was unloaded throughout SiLs' lamina but lowly-detectable in SoLs' CCs (Fig.1a; Imlau et al., 1999; Stadler et al., 2005; Truernit and Sauer, 1995). The SUL-siRNA activity- and free-GFP movement- patterns were similar, with two exceptions. Firstly, the former was more vein-restricted in SiLs than was the latter (Fig.1a), likely reflecting SUL-siRNA consumption. Secondly, whereas both SoLs and SiLs displayed similarly intense vein-centred bleaching, free-GFP was strictly CCrestricted in SoLs (Fig.1a and S1). It appears, therefore, that SUL-RNAi spreads preponderantly into SiLs via long-distance transport and diffusion of siRNAs mainly produced in SoLs. Additionally, a mechanism apparently allows vasculature-tomesophyll movement of siRNAs, but not of free-GFP, in SoLs. 


\section{PD occlusion in SoLs' companion cells suffices to abrogate silencing in SiLs}

The above-described SUL-silencing pattern would predict that targeted PD-occlusion in the SoLs' CCs should suffice to impede bleaching in SiLs. We utilized the inducible dominant-negative iCals3m system (Vatén et al., 2011) to deposit callose at PDs' neck regions in a CC-restricted manner. pSUC2::XVE:iCals3m(iSC)xSS and control SS seedlings were grown until full emergence of primary leaves (L1-L2), then transferred to B-estradiol or DMSO (mock). Since mock- and iCals3m-induced iSCXSS seedlings displayed similar growth-rates (Fig. S2a), we compared RNAi movement at the L6-L7 stage. Bleaching was strongly reduced/abrogated in L3-L7 SiLs of iCals3m-induced iSCXSS plants (Fig. 1b and S2a), coinciding with substantial callose deposition in cotyledons and L1-L2 veins, but only background staining in L4L7-veins (Fig. 1c and S2b). L3 sink-to-source transition leaves occasionally displayed partial callose deposition (Fig. S2b). These patterns agree with pSUC2 being mostly active in SoLs (L1+L2), not SiLs (L4-L7; Fig. 1a). All mock-treated plants only displayed background staining of callose (Fig.1c). The Cals3m and SUL mRNA levels across tissues (SoLs versus sinks [SiLs and roots]), genotypes and treatments concurred with the aforementioned phenotypes (Fig. 1d). DCL4 and SUC2 mRNA levels were unchanged in either sinks or SoLs of iSCXSS plants regardless of treatments, ruling out that reduced SUL-siRNA accumulation underlies impaired photobleaching (Fig. S2c). While biological variation caused total SULsiRNA levels to fluctuate slightly between iSCXSS plants, SUL-siRNA levels were consistently higher ( 40\%) in source- than in sink-tissues upon iCals3m induction compared to mock treatment (Fig. 1e).

That SUL-siRNA retention in iCals3m-induced SoLs correlates with loss-ofsilencing in SiLs supports a role for PDs at the SoLs' CC-SE interface. It also 
suggests that SUL-siRNAs synthesized within the CCs of SoLs contributes the bulk of mobile RNAi in whole plants. Upon phloem-based long-distance transport, their passive unloading likely endows consumption-coupled PTGS in SiLs. Noteworthy, silencing was decreased in most SoLs $(\mathrm{L} 1+\mathrm{L} 2)$ of iCals3m-induced iSCXSS plants, suggesting that the mechanism controlling siRNA-, but not free-GFP- movement outside the SoLs' vasculature is PD-occlusion-sensitive in, at least, the CCs.

\section{Stem-based long-distance transport of RNAi is accompanied by minimal outflow}

Both pSUC2-derived SUL-siRNAs and -artificial miRNAs (pSUC2:amiRSUL) were remarkably efficiently graft-transmitted into non-transgenic rootstocks (Fig.2a; (Brioudes et al., 2021; Devers et al., 2020)). Strikingly, however, photobleaching is conspicuously absent in stems in both systems (Fig. S3) and in stems of the phenotypically analogous JAP3 line based on pSUC2-driven expression of a PHYTOENE DESATURASE(PDS)-derived IR transgene (Fig.2b; (Smith et al., 2007). This lack-of-silencing in stems is striking because potent pSUC2 activity in those tissues (Stadler and Sauer, 2019) enables retrieval of apoplastic photoassimilates potentially leaked during source-to-sink transport (Ayre et al., 2003). Aiding the photoassimilates' long-distance transport is the well-documented symplasmic isolation of the SE-CC-complex(SECCC) in stems, where few and low-conductivity PDs remaining at the CC-phloem parenchyma(PP) interface minimize photoassimilates' dispersion from the inner vascular cylinder to outer-stem tissues (Kempers and van Bel, 1997; Kempers et al., 1998). To explore the idea that si/miRNA movement might be similarly restricted in pSUC2-based silencing systems, we used recombinant Tobacco rattle virus engineered with a PDS fragment (TRV- 
PDS), which yields photobleaching in leaves owing to virus-induced-gene-silencing (Ratcliff et al., 2008). Because viral movement proteins (MPs) actively enlarge PDs' aperture including at the stems' CC-PP-interface (Itaya et al., 2002), we anticipated that TRV-PDS-infected stems would display extensive photobleaching, which was indeed the case (Fig. 2b). These results support the notion that the SE-CC's isolation efficiently limits si/miRNA outflow and, hence, dilution, during their stem-based longdistance transport. This possibly explains the graft-transmission's efficacy and potent systemic effects of SUL-siRNAs despite their mostly SoL-based production (Fig. 2a).

\section{Differential AGO-mediated consumption across vein-distal incipient tissues} likely causes unequal siRNA-length representations in micro-grafted recipient tissues

Both the 21-nt and 24-nt SUL-siRNA species were graft-transmitted into rootstocks (Fig. 2a), contrasting with a previously-established bias towards 24-nt siRNA longdistance mobility (Molnar et al., 2010). To test if differential AGO-mediated consumption between experimental systems could explain this discrepancy, we engineered pATML1::SUL(AS) Arabidopsis, in which the epidermis-specific promoter ATML1 drives the SS-IR. AS leaves accumulate equal amounts of $21-\mathrm{nt}$ and $24-\mathrm{nt}$ SUL-siRNAs but display laminal surface-bleaching likely reflecting SUL-siRNA movement from the epidermis to the lower, photosynthetically-active mesophyll (Fig. 2c). In contrast to $S S$, where CC-produced siRNAs are directly delivered into SEs, more consumption would be expected for AS-derived siRNAs during their multi-celllayer epidermis-to-SE movement via the mesophyll (Fig. 2c-d). Indeed the vasculature remains green in $A S$ leaves (Fig. 2c). Accordingly, and unlike the relatively unbiased transmission of SS-derived 21- and 24-nt SUL-siRNAs (Fig.2a; 
Devers et al., 2020), AS-derived 24-nt SUL-siRNAs predominated in micro-grafted rootstocks (Fig. 2e), evoking the previously-reported 24-nt siRNA bias (Molnar et al., 2010). This suggested a strong and selective consumption of the AS-derived 21-nt SUL-siRNAs in silencing-emitting scions. Accordingly, the transcript of AGO4 (loading the 24-nt SUL-siRNAs; Devers et al., 2020) is $\sim 3$ and $\sim 7$ times less abundant that of AGO1 (loading the 24-nt SUL-siRNAs Devers et al., 2020) in Arabidopsis whole leaves and mesophyll, respectively (Fig. S4). Thus, the 24-nt siRNA bias in the $A S$ system most likely reflects high and selective consumption of 21-nt siRNAs rather than selectivity in long-distance movement per se. The results also support the expected, albeit so-far-untested notions that (i) sRNA size, in addition to 5'-nucleotide identity, strongly influences AGO-mediated consumption, and that (ii) consumption affects not only cell-to-cell but also long-distance vascular movement.

\section{dc/234 rootstocks are suboptimal RNAi-recipient tissues in micro-grafted}

\section{Arabidopsis}

By micro-grafting $S S$ scions onto siRNA-deficient dcl234 rootstocks, we could directly compare the shoot-to-root spread of SUL-siRNAs with that of endo-siRNAs. These included the DCL2/DCL3-dependent 22-nt/24-nt siRNAs from the IR71 singlelocus (Henderson et al., 2006) and two TE/repeat-derived-species (rep2,siRNA1003; Fig. 3a). All siRNAs moved into micro-grafted dcl234 rootstocks, albeit to varying extents (Fig. 3a-b;(Devers et al., 2020)). SUL-siRNA- was stronger than IR71-siRNA graft-transmission, likely reflecting higher production levels of the former. Nonetheless, the 22-nt and 24-nt IR71-siRNAs accumulated equally in dcl234 rootstocks, suggesting limited consumption. This was consistent with IR71, like $S S$, 
but unlike $A S$, being expressed within the leaves' vasculature of RNAi-emitting scions (Devers et al., 2020). Both rep2 and siRNA1003 siRNAs accumulated efficiently in dcl234 rootstocks, contrasting with previous deep-sequencing showing a very low (0-33\%) shoot-to-root transmission of these and additional 24-nt endosiRNAs (Molnar et al., 2010, reanalysed in Fig. S5). Sequencing biases aside, the phloem flow was perhaps better restored under our micro-grafting conditions. Alternatively, AGO4/AGO6 destabilization due to lack-of-cargoes in siRNA-deficient dcl234 recipient rootstocks - as originally reported in rdr2 and nrpd1a (Havecker et al., 2010; Li et al., 2006) - was possibly less pronounced as was, perhaps, its reciprocal consequence, i.e. the enhanced turnover of non-loaded 24-nt siRNAs (Havecker et al., 2010; Smibert et al., 2013; Vaucheret et al., 2006). We considered these possibilities noteworthy because AGO-loaded species contributes mainly to siRNA deep-sequencing results (Grentzinger et al., 2020).

Consistent with AGO4 reduced accumulation impairing siRNA detection in micro-grafted dcl234 recipient tissues, AGO4 levels were partially rescued in rootstocks of WT/dcl234 versus dcl234/dcl234 micro-grafted plants (Fig. 3b). Thus, upon translocation into dcl234 rootstocks, shoot-derived 24-nt siRNAs had likely partially stabilized AGO4 via loading. Accordingly, rep2 and 1003 siRNAs were readily detected in AGO4 IPs performed in rootstocks of WT/dcl234 unlike dcl234/dcl234 plants (Fig. 3b). The AGO4-destabilization's impact of dc/234 was revealed by activity-measurements of graft-transmitted rep2 siRNAs (Fig. 3c). Methylation at rep2 was reduced from $75.64 \%$-to-58.33\% and from $44.23 \%$-to$27.55 \%$ at $\mathrm{CHG}$ and $\mathrm{CHH}$ sites, respectively, in WT/WT versus dcl234/dcl234 grafted rootstocks (Fig. 3c). siRNAs from WT shoots grafted onto dcl234 rootstocks (WT/dcl234) rescued CHG- and $\mathrm{CHH}$-methylation (Fig. 3c). As expected, CG- 
methylation remained unchanged, consistent with its siRNA-independent maintenance (Fig. 3c; Xie et al., 2004). Coincident AGO4 re-stabilization and DNA methylation-rescue confirm functional movement of 24-nt endo-siRNAs, yet at levels and with activities likely underestimated by use of suboptimal and bias-inducing dcl234 recipient tissues owing to an artefactual paucity of AGO4-clade RdDMeffectors. Without altering their graft-transmission per se, this caveat probably decreases detectability of low-to-moderately abundant mobile 24-nt siRNAs.

\section{Stress-induced shootward movement of endo-siRNAs in micro-grafted}

\section{Arabidopsis}

In reverse-grafting, SUL-siRNAs moved from $S S$ transgenic rootstocks to WT shoots, albeit less efficiently than from shoot-to-root (Fig. 3a and 4a). Deepsequencing confirmed this observation (Devers et al., 2020; Fig. S6a) reminiscent of similar transmission-deficits of transgene-derived and endo-siRNAs under similar root-to-shoot micro-grafting settings (Molnar et al. in 2010). To explore a comparable endo-siRNA transmission-deficit without the AGO4-destabilization effect of dc/234, we exploited an IR71 T-DNA knockout (ir71) essentially eliminating IR71-siRNAs (Fig. 4b and S6b); these are near-identical to those of SS in their vasculatureproximal production, mobile attributes and RDR-independency (Devers et al., 2020). Like SUL-siRNAs, IR71-siRNAs were at northern detection limit in shoots of ir71/WT grafts (Fig. 4c, control) likely due to suboptimal root-to-shoot photoassimilates' fluxes incurred by micro-grafting. We thus tested if this caveat could be overcome by increasing IR71-siRNA production through stress application. Among several stresses tested, heat-shock (20 hrs at $37^{\circ} \mathrm{C}$; Fig. $4 \mathrm{~d}$ ) caused a $\sim 4$-fold increase in 
IR71 promoter activity (Fig. 4e). In side-by-side experiments, HS applied under otherwise identical conditions to those of mock-treated plants caused detectable accumulation of 22-nt and 24-nt IR71-siRNA in recipient ir71-shoots (Fig. 4c, $37^{\circ} \mathrm{C}$ ). Therefore, by stimulating IR71-siRNA production/mobility, HS could overcome a major micro-grafting limitation.

\section{Mobile IR71-siRNAs mediate cis-methylation and negatively regulate dozens of transcripts linked to systemic acquired resistance in intact plants}

As much as the physiological relevance of grafting to (endo)-siRNA mobility is debated (Kehr, 2013; Liang et al., 2012) so too is the biological role, if any, of noncell autonomous silencing mediated by these molecules in intact (i.e. non-grafted) plants, aside from TAS3 siRNA cell-to-cell movement during leaf polarization (Chitwood et al., 2009; Schwab et al., 2009). To address this key issue we exploited our previous findings that (i) $I R 71$ is vascularly-expressed and (ii) mobile $I R 71$ siRNAs can be physically isolated, from the lower epidermis located several celllayers away ((Devers et al., 2020); Fig. 5a). We demonstrated on multiple occasions the tissue-specific mechanical separation including in the case of IR71 (Brioudes et al., 2021; Brosnan et al., 2019; Devers et al., 2020). Bisulfite sequencing of IR71 DNA from the incipient vasculature revealed high levels of CG $(80 \%)$ and moderate levels of $\mathrm{CHG}$ and $\mathrm{CHH}$ methylation ( $50 \%$ and $\sim 10 \%$ respectively; Fig. $5 \mathrm{~b})$. By contrast, methylation was markedly reduced at asymmetric, RdDM-dependent cytosines in both vasculature and distal epidermis of ir71 mutant leaves (Fig. 5b). Furthermore, HS treatments slightly increased IR71-siRNA movement and 
asymmetric cytosine methylation in the epidermis (Fig.5a and c). Therefore, mobile IR71-siRNAs mediate RdDM in cis in intact plants.

To explore the global trans-regulatory impact of IR71-siRNAs - encompassing both PTGS and TGS levels - under native (i.e. non-grafted) conditions, we conducted whole-transcriptome analyses of Meselect-isolated epidermis of ir71 vs WT leaves to focus solely on the locus' non-autonomous regulatory effects. In highstringency analyses (FDR cut-off $\leq 0.01) 427$ and 498 transcripts were respectively up- and down-regulated in the ir71 vs WT epidermis (Fig. 5d). GO-term-enrichment of up-regulated mRNAs identified processes strikingly dominated by pathogens' recognition and salicylic acid (SA) biosynthesis/regulation/signalling (Fig. 5e). SA production is triggered during plant-biotroph incompatible interactions following a respiratory burst culminating in the "hypersensitive response" (HR). SA accrues not only within and around HR lesions, but also in distant, non-infected tissues where resistance to secondary infection is primed via "systemic acquired resistance" (SAR; Fu and Dong, 2013). By contrast, processes of biosynthesis/signalling by the oxylipin jasmonic acid (JA) -conferring resistance to necrotrophs and herbivores/woundingprevailed in the down-regulated mRNA set (Fig. 5f). This agrees with SA vs JA signalling being reciprocally antagonistic (Pieterse et al., 2009) and provides the first comprehensive view of gene expression changes caused solely by mobility of a genetically-tractable endo-siRNA cohort in intact plants. Given its large, yet specific mobile regulatory output, $I R 71$ therefore displays attributes of a non-cell autonomous modulator of SAR. 


\section{DISCUSSION}

\section{Systemic RNAi in pSUC2-based systems is the manifestation of mostly a single movement process}

We conclude from our study that mobile RNAi in young tissues of SS and related pSUC2-based systems (Smith et al., 2007; Uddin et al., 2014) reflects diffusion of an siRNA-pool produced within, and translocated from, mature tissues, rather than active and localized cell-to-cell movement in young tissues. Callose deposition in SoLs' CCs indeed suffices to abrogate RNAi-movement in SS plants (Fig. 1). As highlighted by (Paterlini et al., 2021), high callose accumulation within iCals3mexpressing CCs could, in principle, also interfere with apoplastic transport. Under the iCals3m-setting, however, sucrose/trehalose-6-phosphate transport was unaffected (Paterlini et al., 2021), and, likewise, plant growth-rates did not differ here between treatments/genotypes (Fig. S2a). Therefore, PDs are likely central to source-to-sink RNAi translocation, primarily at the SoLs' CC-SE interface. This rationalizes why Cadmium treatment in SoLs inhibited long-distance but not cell-to-cell transgene silencing (Ueki and Citovsky, 2002) since heavy metals induce callose deposition specifically in vasculature-associated cells (Ueki and Citovsky, 2005). Upon phloem unloading, the large PD size-exclusion-limit in SiLs (Stadler et al., 2005) would accommodate passive diffusion of translocated $\sim 13-15 \mathrm{kDa}$ siRNAs (21-nt duplexes) well-below the free-GFP's $27 \mathrm{kDa}$. RNAi movement, at least in pSU2-based systems, is thus the likely manifestation of predominantly vasculature-dependent long-distance siRNA transport, as indeed previously anticipated (Stadler and Sauer, 2019). 


\section{Differential AGO-mediated consumption of siRNAs in distinct experimental settings likely explains an apparent size-selectivity in their mobility}

Free-GFP invades the near-entirety of SiLs' laminas, reflecting that no conceivable plant-based mechanism might hinder this xenobiotic protein's spread. AGO-siRNA co-evolution, by contrast, likely underpins the vein-restricted SUL-siRNA activitypattern in SiLs. Of the 21-nt and 24-nt species only the former mediates mobile PTGS/photobleaching in an AGO1-(5'U) and, secondarily, AGO2-(5'A)-dependent manner (Devers et al., 2020). Of the two, AGO1-based consumption likely prevails due to its overrepresentation in leaves (Fig. S4). Accordingly, genetically reducing AGO1 loading-efficacy in silencing-traversed cells suffices to extend 5'U-si/miRNA physical movement throughout leaves (Brioudes et al., 2021; Devers et al., 2020). Loaded into the lesser abundant AGO4, a higher proportion of 24-nt SUL-siRNAs would likely move beyond the vein-restricted activity-pattern of their 21-nt counterparts. This would be undiagnosed, however, as they do not contribute PTGS/photobleaching (Dunoyer et al., 2005). In the AS setting, multiple cell-layers separating incipient epidermis from recipient SEs likely exacerbate the differential AGO1 versus AGO4 consumption, rationalizing how 24-nt SUL-siRNAs are nearexclusively detected in graft-recipient tissues (Fig. 2e). Consistent with the above observation, an artificial $A T M L 1$-driven 5'U-terminal miRNA displayed no apparent vascular activity unless expressed at extremely high levels presumably overcoming its AGO1-mediated consumption across epidermis-to-vasculature-traversed tissues (Brioudes et al., 2021; Skopelitis et al., 2018).

Consumption also resolves the long-standing conundrum as to why 24-nt siRNAs appear selectively correlated with long-distance mobility in some, unlike other, experimental systems. In GFP-transgenic N.benthamiana, Agrobacterium- 
based transient expression of silencing-inducing constructs initiate mobile RNAi. Both 21-nt and 24-nt GFP-siRNAs accumulate mostly in the agroinfiltrated patch's epidermis and mesophyll (Hamilton et al., 2002) where high AGO1 levels (Fig. S4) and thus strong AGO1-based consumption likely prevents 21-nt GFP-siRNAs from reaching the vasculature. This likely explains why 24-nt GFP-siRNAs, presumably far-less AGO4-consumed, positively correlate with systemic silencing in this system (Hamilton et al., 2002). The strong 24-nt siRNA bias observed genome-wide in the micro-grafting studies of Molnar et al. (2010) presumably also reflects that only a subfraction of the total pool of 21-nt endo-siRNA species is sufficiently vasculatureproximal to evade significant consumption by AGO1. Indeed, an siRNA-length bias was not observed in three-out-of-three independent systems studied in Devers et al. (2020), all of which were artificially (SS) or naturally (polerovirus and $I R 71$ ) poised to trigger mobile RNAi in the direct SEs' vicinity, thereby mostly bypassing AGO1mediated consumption. Thus, what might be interpreted as selectivity/conditionality based on mobile siRNA-size most likely reflects system-intrinsic differences influencing the extent of differential AGO-mediated consumption. From a natural standpoint, these findings reinforce the previously-made suggestion (Skopelitis et al., 2018) that efficient long-distance movement would be an attribute of si/miRNAs naturally expressed close to, or within, the vasculature, as are indeed all systemic miRNAs reported so far in plants (Brioudes et al., 2021; Brosnan et al., 2019; Lin et al., 2008; Pant et al., 2008).

\section{High efficiency of long-distance RNAi transport}


It has been proposed that vascular miRNAs might only display efficient non-cell autonomous effects if their activity is fostered, in recipient tissues, by "transitivity", whereby mobile secondary siRNAs are processed from long dsRNA amplified by RNA-DEPENDENT-RNA-POLYMERASES(RDRs) from cleaved miRNA-target transcripts (Skopelitis et al., 2018). However, long-distance movement of miR399 (Lin et al., 2008; Pant et al., 2008), miR2111 in Lotus japonicus (Tsikou et al., 2018) and amiRSUL (Brioudes et al., 2021) is not accompanied by secondary siRNA production. We proposed, similarly, that primary i.e. non-RDR-amplified RNAi might suffice for robust long-distance siRNA-mediated silencing (Devers et al., 2020), even though it had been so far impossible to disrupt, in real time, ongoing RNAi in planta, a necessary approach to measure the process' potency and persistence. Our ability to interrupt SS-silencing via targeted callose deposition now reveals that transport initiated in only 2-3 SoLs is sustained, without RDR amplification, in at least four SiLs. Additionally, none-of-three pSUC2-based si/miRNA silencing systems displayed stem photobleaching in contrast to the viral-based TRV-PDS-infected stems. This suggests that symplasmic isolation of stem SE-CC complexes -which is disrupted by virus-encoded MPs- ensures optimal long-distance transmission of mobile si/miRNAs by limiting/precluding their dilution in stems. This efficacy, in turn, rationalizes why transitivity is dispensable in recipient sink tissues, in at least the aforementioned mi/siRNA systems and during cross-kindom (Subhankar et al., 2021). We note, however, that SUL silencing in SiLs likely requires a continued RNAi input because residual SUL-siRNAs in SiLs failed to promote bleaching after PD closure in SoLs (Fig. 1). Thus, transitivity might primarily enable silencing sustainability over prolonged periods of time. This might be particularly relevant during (often life-long) viral infections (Pumplin and Voinnet, 2013) or extended 
developmental processes such as leaf polarization (Chitwood et al., 2009; Schwab et al., 2009). By contrast, transitivity may not be a desirable feature of short-term sRNA-based responses requiring rapid reversal, for instance upon the elapse of a stress.

\section{Possible active forms of cell-to-cell RNAi transport}

PD closure at the CC-SE interface reduced bleaching not only in the signal-recipient SiLs, but also in the signal-incipient SoLs in which, therefore, SUL-siRNAs must also move via PDs toward the mesophyll at the CC-PP-bundle-sheath interface. Similar movement is observed in pSUC2-based artificial miRNA systems, including amiRSUL, though the involvement of PDs has not been experimentally verified in these cases (Brioudes et al., 2021; Skopelitis et al., 2018). In sharp contrast, free GFP remains strictly confined within the CCs in SoLs (Fig. 1a and S1; (Brioudes et al., 2021; Skopelitis et al., 2018), in line with its retention in phloem cells where only apoplastic transport occurs (Stadler et al., 2005; Werner et al., 2011). Thus, a mechanism exists in SoLs, that propagates si/miRNAs outside the CCs against the photoassimilates' flux in a manner not permitting free GFP movement. Moreover, this process must occur, at least partly, at the CC-PP interface in which low-conductivity PDs occur at a low frequency, incidentally explaining the prevailing apoplastic phloem-loading of Arabidopsis (Turgeon, 1996). Therefore, the CC-to-PP sRNA movement in Sols is perhaps the manifestation of an active PD-based process, unlike movement seen at most other cellular junctions involved in pSUC2-based RNAi systems. Active transport of endogenous/viral proteins likely involves sophisticated interactions at the cytosolic side of the desmotubule, a compressed cortical ER segment linking adjacent cells through the PD lumen (Beachy and 
Heinlein, 2000; Kim et al., 2005). Strikingly, PD-based free GFP movement is desmotubule-independent (Oparka et al., 1999). Other, perhaps unique sRNAintrinsic features could underpin their crossing of various PD-based boundaries as part of a potentially dedicated transport mechanism, including their negative charge if one assumes that si/miRNAs move as protein-free entities.

\section{Genetically tractable, discrete loci enable studies of endo-siRNA movement and functions in intact plants and perhaps over generations}

Long-distance endo-RNAi studies involving grafted dcl234 recipient tissues concluded that only a third of all Arabidopsis 24-nt siRNA-generating loci apparently spawn mobile species (Lewsey et al., 2016; Molnar et al., 2010), providing further possible evidence of selective siRNA mobility (Maizel et al., 2020). Recent work argues against this notion in cell-to-cell movement settings, however (Devers et al., 2020), and we further suggest here that all siRNAs have a graft-transmission potential. Yet, the dramatic albeit artefactual loss-of-AGO4 stability suggests that, for them to be detected in dcl234-receiving-rootstocks, siRNAs should be sufficiently abundant to stabilize their effectors and, reciprocally, for those effectors to stabilize their cargoes. Consequently, the amount, diversity and breath-of-activities of mobile endo-siRNAs have been likely underestimated in siRNA-deficient dcl234 recipient tissues. Use of this now-apparent suboptimal background has been mandated by the domination, in movement studies, of TE/repeat-derived siRNAs (Lewsey et al., 2016; Molnar et al., 2010) whose genome-multiplicity makes single-loci knockouts de facto unavailable.

IR71 illustrates how discrete, genetically-tractable sources of mobile endoRNAi bestow the use of WT recipient-tissues to overcome dc/234-associated 
caveats, and how stress-induced endo-RNAi modulation can bypass unidirectionality caused by micro-grafting. The naturally vasculature-restricted $I R 71$ expression allowed mobile endo-RNAi study in intact plants by avoiding the contentious process of (micro)grafting altogether (Kehr, 2013). Mechanical tissue-separation uncovered a cell-to-cell, in addition to long-distance, component to IR71-siRNA mobility, revealing transgenic RNAi-like properties (Fig. 5; Devers et al., 2020). IR71-siRNA cell-to-cell spread elicits RdDM, at least in cis, and trans-regulates dozens of mRNAs collectively promoting SAR via salicylate biosynthesis/signalling/metabolism (Pieterse et al., 2009). Conversely, downregulated (in all likelihood indirectly) mRNAs in ir71 plants are biased toward the SA-antagonistic JA pathway (Pieterse et al., 2009). Its basal expression and non-autonomous action in healthy plants thus suggest that $I R 71$ could potentially act as a rheostat enabling rapid, reversible tuning of JA vs SA responses not achievable by separate transcriptional control of possibly dozens of discrete loci. The HS induction of $I R 71$ might also illustrate a trade-off for resource allocation between abiotic vs biotic stress adaptation (Berens et al., 2019).

We failed to identify the most upstream regulatory nodes - and thus the likely direct targets - of the IR71-controlled gene network. Indeed, transcriptome mining coupled to target predictions were not backed-up by degradome-based searches for AGO:sRNA-mediated slicing events. We note the unusual preponderance of 22-ntlong, instead of 21-nt-long, PTGS-inducing IR71-siRNAs. Recently, stress-induced 22-nt siRNAs were found to mostly incur translational repression of predicted mRNA targets in Arabidopsis (Wu et al., 2020). A similar effect from IR71-siRNAs would not have been diagnosed by transcriptomics reporting, perhaps, mere secondary consequences of dysregulating, at the translation level, a narrower subset of primary $I R 71$ targets. Regardless, and while more work is needed to assess IR71's potential 
roles in SAR/stress regulation, our findings now provide a tangible framework for functional mobile endo-RNAi studies in Arabidopsis. The HS-induction of IR71siRNA and their ensuing shootward movement in ir71/WT micro-grafting might also facilitate transgenerational epigenetic inheritance studies from this geneticallytractable locus.

\section{ACKNOWLEDGMENTS}

We thank Voinnet lab members for fruitful discussions, A. Imboden for plant care, $E$ Truernit for providing pSUC2::tmGFP9 and pSUC2::GFP seeds, C. Himber for selecting homozygous $p A T M L 1:: S U L-I R$ lines, S. Mirlohi and G. Schott for some images and the TRV-PDS infections, and the ETH ScopeM unit for providing the microscopy facility. This work was supported by an EMBO Long-Term Fellowship (ALTF 728-2009) to C.A.B., a Marie Curie Intra-European Fellowship for career development (FP7-PEOPLE-IEF; number 623826) to E.A.D. and a European Research Council advanced grant (Frontiers of RNAi-II; number 323071) to O.V.

\section{AUTHORS CONTRIBUTIONS}

C.A.B., E.A.D., and O.V. designed the project and all experiments. C.A.B. conducted all experiments relating to $S S$ grafting, MeSelect and IR71. E.A.D. conducted experiments relating to PD and iCals $3 m$-induced callose deposition and $A S$ grafting. A.S. performed all bioinformatic analysis. P.L. assisted in molecular analysis of $I R 71$. S.L. and Y.H contributed the original pSUC2-based iCals3m system. C.A.B., E.A.D. and O.V. analyzed the data. C.A.B. and E.A.D. assembled all of the figures and, together with O.V., wrote the manuscript. All authors read and approved the manuscript. 
bioRxiv preprint doi: https://doi.org/10.1101/2021.10.06.463290; this version posted October 6, 2021. The copyright holder for this preprint (which was not certified by peer review) is the author/funder. All rights reserved. No reuse allowed without permission. 
Figure 1. CC-to-SE translocation of the SS-silencing signal occurs via plasmodesmata. (a) Seedlings grown $1 / 2$ MS containing agar plates expressing the insoluble pSUC2::tmGFP9, the soluble pSUC2::GFP or the RNAi source SS. Left and middle panel show fluorescence images of the respective plants. Arrowheads point to vein restriction of fluorescent signal in source leaves. Scale bar $5 \mathrm{~mm}$. For the phenotype of a soil grown SS plant, see figure 2 and $S 1$. (b) 14 days old seedlings of SS and SS $x$ iSC grown 7dpg on either DMSO (mock) or $20 \mu \mathrm{M} 17-\beta-$ estradiol containing media. See also figure S2a. (c) Epifluorescence microscopy analysis of callose deposition after aniline blue staining of plants taken from the experiment shown in A. Scale bars represent $200 \mu \mathrm{M}$. See also figure S2b. (d) qRTPCR analysis of Cals3m and SUL. Black bars, SS; grey bars, iSC. Error bars represent $S E, n=6-10$. Asterisks indicate statistically significant differences $(p \leq 0.05$, two-tailed Student's t-test with equal variance [F-test, $p>0.05]$ ). (e) RNA gel blot analysis of source (SoLs; Cotyledons, L1-L2) and sink (L-3-7, roots) tissues from individual SS and iSC plants shown in (b). U6 serves as a loading control.

Figure 2. Consumption, initiating expression domaining and PD architecture dictate the mobile nature siRNA species. (a) RNA gel blot analysis of biological duplicates of SS shoot grafted to WT root. U6 serves as a loading control. (b) Images of equivalent age rosette stage (bottom panels) and flowering (top panels) WT (left) and JAP3 (middle panels) plants. Right panel shows TRV-PDS infected flowering plant. Insets show distinctive lack or presence of bleaching in stem tissues. (c) Images of whole plant showing photobleaching phenotypes of WT and AS lines. (d) Cross section of an Arabidopsis leaf showing the distance siRNAs would have to travel from adaxial or abaxial sides to initiate vasculature-based graft transmissible 
silencing (red arrow). The expression domain of ATML1 is exclusively located in the epidermis. Abbreviations: as, air space; bs, bundle sheath; ep, epidermis; ph, phloem; pm, palisade mesophyll; sm, spongy mesophyll; xy, xylem. (e) RNA gel blot analysis of shoots (left; lane 1-4) and root (right; lane 6-9) of indicated graft combinations. Black triangle indicates increase in exposure for the SUL probe. miR160 and U6 serve as loading controls.

Figure 3. Graft-transmissible endo-siRNA movement, limiting factors and functionality. (a) RNA gel blot analysis of the indicated graft combinations looking at either transgene- (SS) or endo-siRNA movement to a WT or dcl234 root. U6 serves as a loading control (b) Immunoprecipitation of AGO4 in the indicated graft combinations. Top panels represent western blot analysis of AGO4 protein levels Input (Left) and IP (Right). Coomassie Blue (CB) serves as a loading control. Lower panels represent RNA gel blot analysis of the same immunoprecipitations. U6 serves as a loading control (c) Bisulfite sequencing of rep2 loci in the of the root of the indicated graft combinations. Graphs depict averages with error bars showing 95\% Wilson score confidence intervals. Numbers and distribution are shown in Table S1.

Figure 4. Heat shock inducible root-to-shoot movement of IR71 derived endo-

siRNAs. (a) RNA gel blot analysis of Root (left) and Shoot (right) of SS root grafted to WT shoots. U6 serves as a loading control. (b) siRNA profiles of IR71 loci in WT and ir71 mutant lines (see also figure S5b). Upper panels show the profile and size distribution (right) of IR71-derived siRNAs in WT plants. Lower panel shows siRNA profile of IR71-derived siRNAs in T-DNA line (ir71 -/-). RPM - Reads per Million, colour code: Blue - 20-21-nt, Green - 22-23-nt and Red - 24-25-nt. (c) RNA gel blot 
analysis of inflorescence tissue under heat shock (HS) conditions for the indicated time. U6 serves as a loading control. (d) qRT-PCR analysis of heat shocked plants expressing a pIR71::GUS reporter. Error bars represent +/- SD of 3 biological replicates. (e) RNA gel blot analysis of indicated graft combinations from either the root or scion of IR71-derived siRNAs under control or heat-shock conditions.

Figure 5. Functionality of endogenous inverted-repeat derived siRNAs under native conditions. (a) RNA gel blot analysis of IR71-derived siRNAs from the either whole leaf, vasculature (Vasc) or epidermis (Epi) under control (left) or heat shock (right; $37^{\circ} \mathrm{C}$ ). miR159 and U6 serve as controls. (b) Bisulfite sequencing analysis of IR71 locus from the vascular (Vasc) and epidermal (Epi) tissue in either WT or ir71 mutants. Graphs depict averages with error bars showing 95\% Wilson score confidence intervals. Numbers and distribution are shown in Table S1. (c) Bisulfite sequencing analysis of $I R 71$ locus from epidermal tissue either Wildtype (Col) or ir71 mutants under normal or heat shock $\left(37^{\circ} \mathrm{C}\right)$ conditions. Graphs depict averages with error bars showing 95\% Wilson score confidence intervals. Numbers and distribution are shown in Table S2. (d) MA-plot representing the result of mRNA-seq differential analysis between epidermal tissue of WT or ir71 mutant. The number of genes considered as significantly up- or down-regulated (red dots; FDR $<0.01$ ) are shown on the top- or bottom-right. (e) Top ten GO-term enrichments of the 427 upregulated genes. (f) Top ten GO-term enrichments of the 498 downregulated genes. 


\section{References}

Ayre, B.G., Keller, F., and Turgeon, R. (2003). Symplastic continuity between companion cells and the translocation stream: long-distance transport is controlled by retention and retrieval mechanisms in the phloem. Plant Physiol 131, 1518-1528.

Beachy, R.N., and Heinlein, M. (2000). Role of P30 in replication and spread of TMV. Traffic 1,540544.

Berens, M.L., Wolinska, K.W., Spaepen, S., Ziegler, J., Nobori, T., Nair, A., Krüler, V., Winkelmüller, T.M., Wang, Y., Mine, A., et al. (2019). Balancing trade-offs between biotic and abiotic stress responses through leaf age-dependent variation in stress hormone cross-talk. Proc Natl Acad Sci USA 116, 2364-2373.

Bologna, N.G., and Voinnet, O. (2014). The diversity, biogenesis, and activities of endogenous silencing small RNAs in Arabidopsis. Annu Rev Plant Biol 65, 473-503.

Bouché, N., Lauressergues, D., Gasciolli, V., and Vaucheret, H. (2006). An antagonistic function for Arabidopsis DCL2 in development and a new function for DCL4 in generating viral siRNAs. EMBO J. 25, 3347-3356.

Brioudes, F., Jay, F., Sarazin, A., Grentzinger, T., Devers, E.A., and Voinnet, O. (2021). HASTY, the Arabidopsis EXPORTIN5 ortholog, regulates cell-to-cell and vascular microRNA movement. EMBO J e107455.

Brosnan, C.A., Mitter, N., Christie, M., Smith, N.A., Waterhouse, P.M., and Carroll, B.J. (2007). Nuclear gene silencing directs reception of long-distance mRNA silencing in Arabidopsis. Proc. Natl. Acad. Sci. U.S.A. 104, 14741-14746.

Brosnan, C.A., Sarazin, A., Lim, P., Bologna, N.G., Hirsch-Hoffmann, M., and Voinnet, O. (2019). Genome-scale, single-cell-type resolution of microRNA activities within a whole plant organ. EMBO J. 38, e100754.

Chitwood, D.H., Nogueira, F.T.S., Howell, M.D., Montgomery, T.A., Carrington, J.C., and Timmermans, M.C.P. (2009). Pattern formation via small RNA mobility. Genes Dev. 23, 549-554.

Conine, C.C., and Rando, O.J. (2021). Soma-to-germline RNA communication. Nat Rev Genet.

Deleris, A., Gallego-Bartolome, J., Bao, J., Kasschau, K.D., Carrington, J.C., and Voinnet, O. (2006). Hierarchical action and inhibition of plant Dicer-like proteins in antiviral defense. Science 313, 68-71.

Devers, E.A., Brosnan, C.A., Sarazin, A., Albertini, D., Amsler, A.C., Brioudes, F., Jullien, P.E., Lim, P., Schott, G., and Voinnet, O. (2020). Movement and differential consumption of short interfering RNA duplexes underlie mobile RNA interference. Nat. Plants.

Dunoyer, P., Himber, C., and Voinnet, O. (2005). DICER-LIKE 4 is required for RNA interference and produces the 21-nucleotide small interfering RNA component of the plant cell-to-cell silencing signal. Nat. Genet. 37, 1356-1360.

Fu, Z.Q., and Dong, X. (2013). Systemic acquired resistance: turning local infection into global defense. Annu Rev Plant Biol 64, 839-863.

Gasciolli, V., Mallory, A.C., Bartel, D.P., and Vaucheret, H. (2005). Partially redundant functions of Arabidopsis DICER-like enzymes and a role for DCL4 in producing trans-acting siRNAs. Curr. Biol. $15,1494-1500$. 
Grentzinger, T., Oberlin, S., Schott, G., Handler, D., Svozil, J., Barragan-Borrero, V., Humbert, A., Duharcourt, S., Brennecke, J., and Voinnet, O. (2020). A universal method for the rapid isolation of all known classes of functional silencing small RNAs. Nucleic Acids Res 48, e79.

Hamilton, A., Voinnet, O., Chappell, L., and Baulcombe, D. (2002). Two classes of short interfering RNA in RNA silencing. EMBO J 21, 4671-4679.

Havecker, E.R., Wallbridge, L.M., Hardcastle, T.J., Bush, M.S., Kelly, K.A., Dunn, R.M., Schwach, F., Doonan, J.H., and Baulcombe, D.C. (2010). The Arabidopsis RNA-Directed DNA Methylation Argonautes Functionally Diverge Based on Their Expression and Interaction with Target Loci. Plant Cell 22, 321-334.

Henderson, I.R., Zhang, X., Lu, C., Johnson, L., Meyers, B.C., Green, P.J., and Jacobsen, S.E. (2006). Dissecting Arabidopsis thaliana DICER function in small RNA processing, gene silencing and DNA methylation patterning. Nat. Genet. 38, 721-725.

Himber, C., Dunoyer, P., Moissiard, G., Ritzenthaler, C., and Voinnet, O. (2003). Transitivitydependent and -independent cell-to-cell movement of RNA silencing. EMBO J. 22, 4523-4533.

Imlau, A., Truernit, E., and Sauer, N. (1999). Cell-to-cell and long-distance trafficking of the green fluorescent protein in the phloem and symplastic unloading of the protein into sink tissues. Plant Cell $11,309-322$.

Itaya, A., Ma, F., Qi, Y., Matsuda, Y., Zhu, Y., Liang, G., and Ding, B. (2002). Plasmodesma-mediated selective protein traffic between "symplasmically isolated" cells probed by a viral movement protein. Plant Cell 14, 2071-2083.

Kehr, J. (2013). Systemic regulation of mineral homeostasis by micro RNAs. Front Plant Sci 4, 145.

Kempers, R., and van Bel, A.J.E. (1997). Symplasmic connections between sieve element and companion cell in the stem phloem ofVicia faba $\mathrm{L}$. have a molecular exclusion limit of at least $10 \mathrm{kDa}$. Planta 201, 195-201.

Kempers, R., Ammerlaan, A., and van Bel, A.J.E. (1998). Symplasmic Constriction and Ultrastructural Features of the Sieve Element/Companion Cell Complex in the Transport Phloem of Apoplasmically and Symplasmically Phloem-Loading Species1. Plant Physiology 116, 271-278.

Kim, J.-Y., Rim, Y., Wang, J., and Jackson, D. (2005). A novel cell-to-cell trafficking assay indicates that the KNOX homeodomain is necessary and sufficient for intercellular protein and mRNA trafficking. Genes Dev. 19, 788-793.

Kobayashi, K., and Zambryski, P. (2007). RNA silencing and its cell-to-cell spread during Arabidopsis embryogenesis. Plant J. 50, 597-604.

Kundariya, H., Yang, X., Morton, K., Sanchez, R., Axtell, M.J., Hutton, S.F., Fromm, M., and Mackenzie, S.A. (2020). MSH1-induced heritable enhanced growth vigor through grafting is associated with the RdDM pathway in plants. Nat Commun 11, 5343.

Law, J.A., and Jacobsen, S.E. (2010). Establishing, maintaining and modifying DNA methylation patterns in plants and animals. Nat. Rev. Genet. 11, 204-220.

Lewsey, M.G., Hardcastle, T.J., Melnyk, C.W., Molnar, A., Valli, A., Urich, M.A., Nery, J.R., Baulcombe, D.C., and Ecker, J.R. (2016). Mobile small RNAs regulate genome-wide DNA methylation. Proc. Natl. Acad. Sci. U.S.A. 113, E801-810.

Li, C.F., Pontes, O., El-Shami, M., Henderson, I.R., Bernatavichute, Y.V., Chan, S.W.-L., Lagrange, T., Pikaard, C.S., and Jacobsen, S.E. (2006). An ARGONAUTE4-containing nuclear processing center colocalized with Cajal bodies in Arabidopsis thaliana. Cell 126, 93-106. 
Li, S., Wang, X., Xu, W., Liu, T., Cai, C., Chen, L., Clark, C.B., and Ma, J. (2021). Unidirectional movement of small RNAs from shoots to roots in interspecific heterografts. Nat Plants 7, 50-59.

Lin, S.-I., Chiang, S.-F., Lin, W.-Y., Chen, J.-W., Tseng, C.-Y., Wu, P.-C., and Chiou, T.-J. (2008). Regulatory network of microRNA399 and PHO2 by systemic signaling. Plant Physiol 147, 732-746.

Liu, Y., Teng, C., Xia, R., and Meyers, B.C. (2020). PhasiRNAs in Plants: Their Biogenesis, Genic Sources, and Roles in Stress Responses, Development, and Reproduction. Plant Cell 32, 30593080 .

Maizel, A., Markmann, K., Timmermans, M., and Wachter, A. (2020). To move or not to move: roles and specificity of plant RNA mobility. Current Opinion in Plant Biology 57, 52-60.

Mi, S., Cai, T., Hu, Y., Chen, Y., Hodges, E., Ni, F., Wu, L., Li, S., Zhou, H., Long, C., et al. (2008). Sorting of small RNAs into Arabidopsis argonaute complexes is directed by the 5' terminal nucleotide. Cell 133, 116-127.

Molnar, A., Melnyk, C.W., Bassett, A., Hardcastle, T.J., Dunn, R., and Baulcombe, D.C. (2010). Small silencing RNAs in plants are mobile and direct epigenetic modification in recipient cells. Science 328, 872-875.

Oparka, K.J., Roberts, A.G., Boevink, P., Santa Cruz, S., Roberts, I., Pradel, K.S., Imlau, A., Kotlizky, G., Sauer, N., and Epel, B. (1999). Simple, but not branched, plasmodesmata allow the nonspecific trafficking of proteins in developing tobacco leaves. Cell 97, 743-754.

Palauqui, J.C., Elmayan, T., De Borne, F.D., Crete, P., Charles, C., and Vaucheret, H. (1996). Frequencies, Timing, and Spatial Patterns of Co-Suppression of Nitrate Reductase and Nitrite Reductase in Transgenic Tobacco Plants. Plant Physiol. 112, 1447-1456.

Palauqui, J.C., Elmayan, T., Pollien, J.M., and Vaucheret, H. (1997). Systemic acquired silencing: transgene-specific post-transcriptional silencing is transmitted by grafting from silenced stocks to nonsilenced scions. EMBO J. 16, 4738-4745.

Pant, B.D., Buhtz, A., Kehr, J., and Scheible, W.-R. (2008). MicroRNA399 is a long-distance signal for the regulation of plant phosphate homeostasis. Plant $\mathrm{J} 53,731-738$.

Paterlini, A., Dorussen, D., Fichtner, F., van Rongen, M., Delacruz, R., Vojnović, A., Helariutta, Y., and Leyser, O. (2021). Callose accumulation in specific phloem cell types reduces axillary bud growth in Arabidopsis thaliana. New Phytol 231, 516-523.

Pieterse, C.M.J., Leon-Reyes, A., Van der Ent, S., and Van Wees, S.C.M. (2009). Networking by small-molecule hormones in plant immunity. Nat. Chem. Biol. 5, 308-316.

Pumplin, N., and Voinnet, O. (2013). RNA silencing suppression by plant pathogens: defence, counter-defence and counter-counter-defence. Nat. Rev. Microbiol. 11, 745-760.

Ratcliff, F., Martin-Hernandez, A.M., and Baulcombe, D.C. (2008). Technical Advance: Tobacco rattle virus as a vector for analysis of gene function by silencing: TRV-induced gene silencing. The Plant Journal 25, 237-245.

Rosas-Diaz, T., Zhang, D., Fan, P., Wang, L., Ding, X., Jiang, Y., Jimenez-Gongora, T., MedinaPuche, L., Zhao, X., Feng, Z., et al. (2018). A virus-targeted plant receptor-like kinase promotes cellto-cell spread of RNAi. Proc. Natl. Acad. Sci. U.S.A. 115, 1388-1393.

Schwab, R., Maizel, A., Ruiz-Ferrer, V., Garcia, D., Bayer, M., Crespi, M., Voinnet, O., and Martienssen, R.A. (2009). Endogenous TasiRNAs mediate non-cell autonomous effects on gene regulation in Arabidopsis thaliana. PLoS ONE 4, e5980. 
Skopelitis, D.S., Hill, K., Klesen, S., Marco, C.F., von Born, P., Chitwood, D.H., and Timmermans, M.C.P. (2018). Gating of miRNA movement at defined cell-cell interfaces governs their impact as positional signals. Nat Commun 9, 3107.

Smibert, P., Yang, J.-S., Azzam, G., Liu, J.-L., and Lai, E.C. (2013). Homeostatic control of Argonaute stability by microRNA availability. Nat. Struct. Mol. Biol. 20, 789-795.

Smith, L.M., Pontes, O., Searle, I., Yelina, N., Yousafzai, F.K., Herr, A.J., Pikaard, C.S., and Baulcombe, D.C. (2007). An SNF2 protein associated with nuclear RNA silencing and the spread of a silencing signal between cells in Arabidopsis. Plant Cell 19, 1507-1521.

Stadler, R., and Sauer, N. (2019). The AtSUC2 Promoter: A Powerful Tool to Study Phloem Physiology and Development. Methods Mol Biol 2014, 267-287.

Stadler, R., Wright, K.M., Lauterbach, C., Amon, G., Gahrtz, M., Feuerstein, A., Oparka, K.J., and Sauer, N. (2005). Expression of GFP-fusions in Arabidopsis companion cells reveals non-specific protein trafficking into sieve elements and identifies a novel post-phloem domain in roots. Plant J. 41, 319-331.

Subhankar, B., Yamaguchi, K., Shigenobu, S., and Aoki, K. (2021). Trans-species small RNAs move long distances in a parasitic plant complex. Plant Biotechnology 38, 187-196.

Truernit, E., and Sauer, N. (1995). The promoter of the Arabidopsis thaliana SUC2 sucrose-H+ symporter gene directs expression of beta-glucuronidase to the phloem: evidence for phloem loading and unloading by SUC2. Planta 196, 564-570.

Tsikou, D., Yan, Z., Holt, D.B., Abel, N.B., Reid, D.E., Madsen, L.H., Bhasin, H., Sexauer, M., Stougaard, J., and Markmann, K. (2018). Systemic control of legume susceptibility to rhizobial infection by a mobile microRNA. Science 362, 233-236.

Turgeon, R. (1996). Phloem loading and plasmodesmata. Trends in Plant Science 1, 418-423.

Uddin, M.N., Dunoyer, P., Schott, G., Akhter, S., Shi, C., Lucas, W.J., Voinnet, O., and Kim, J.-Y. (2014). The protein kinase TOUSLED facilitates RNAi in Arabidopsis. Nucleic Acids Res. 42, 79717980.

Ueki, S., and Citovsky, V. (2002). The systemic movement of a tobamovirus is inhibited by a cadmium-ion-induced glycine-rich protein. Nat Cell Biol 4, 478-486.

Ueki, S., and Citovsky, V. (2005). Identification of an interactor of cadmium ion-induced glycine-rich protein involved in regulation of callose levels in plant vasculature. Proc Natl Acad Sci U S A 102, 12089-12094.

Vatén, A., Dettmer, J., Wu, S., Stierhof, Y.-D., Miyashima, S., Yadav, S.R., Roberts, C.J., Campilho, A., Bulone, V., Lichtenberger, R., et al. (2011). Callose biosynthesis regulates symplastic trafficking during root development. Dev. Cell 21, 1144-1155.

Vaucheret, H., Mallory, A.C., and Bartel, D.P. (2006). AGO1 homeostasis entails coexpression of MIR168 and AGO1 and preferential stabilization of miR168 by AGO1. Mol. Cell 22, 129-136.

Voinnet, O., and Baulcombe, D.C. (1997). Systemic signalling in gene silencing. Nature 389, 553.

Voinnet, O., Vain, P., Angell, S., and Baulcombe, D.C. (1998). Systemic spread of sequence-specific transgene RNA degradation in plants is initiated by localized introduction of ectopic promoterless DNA. Cell 95, 177-187.

Werner, D., Gerlitz, N., and Stadler, R. (2011). A dual switch in phloem unloading during ovule development in Arabidopsis. Protoplasma 248, 225-235. 
Wu, H., Li, B., Iwakawa, H., Pan, Y., Tang, X., Ling-hu, Q., Liu, Y., Sheng, S., Feng, L., Zhang, H., et al. (2020). Plant 22-nt siRNAs mediate translational repression and stress adaptation. Nature 581, 89-93.

Xie, Z., Johansen, L.K., Gustafson, A.M., Kasschau, K.D., Lellis, A.D., Zilberman, D., Jacobsen, S.E., and Carrington, J.C. (2004). Genetic and functional diversification of small RNA pathways in plants. PLoS Biol. 2, E104. 


\section{Material and Methods}

\section{Plant material, growth conditions}

Wild-type(WT) and mutant Arabidopsis (ecotype Columbia: Col-0) were germinated and grown under long-day light conditions at $22^{\circ} \mathrm{C}$ on $1 / 2$ Murashige and Skoog(MS) medium. The pSUC2::SUL-IR(SS), pIR71::GUS, pSUC2::GFP, pSUC2::tmGFP9, JAP3 and $d c / 2-1 / d c / 3-1 / d c / 4-2$ lines were described previously (Deleris et al., 2006; Devers et al., 2020; Himber et al., 2003; Smith et al., 2007; Stadler et al., 2005). The T-DNA-insertion line for IR71 is SALK_006309. For root isolation, plants were either grown vertically on mesh-covered solid media (Sefear Nitrex 03-65125) or hydroponically (Devers et al., 2020; Gibeaut et al., 1997; Tocquin et al., 2003). For iCals3m experiments the pSUC2::iCals3m(iSC) parental lines "21-1", "23-1" and "251" were tested for callose induction and developmental defects on a 0-100 $\mu \mathrm{M} 17-ß-$ estradiol (Sigma) concentration-range. Since "23-1" and "25-1" showed growth defects at all concentrations, "21-1"(iSC) was selected and crossed to SS. Seeds of iSC or iSCXSS (F3) were germinated and upon ermegence of the first true leaves L1\&L2, 10-15 seedlings were transferred to fresh solid MS containing 20 MM 17-ßestradiol or DMSO (Sigma). For heat-shock experiments, 8 week-old micro-grafted plants were subjected to $37^{\circ} \mathrm{C}$ for $20 \mathrm{hrs}$.

\section{Micrografting}

Micro-grafting was as previously described (Brosnan et al., 2007; Turnbull et al., 2002). Seedlings were germinated on vertical $1 / 2$ MS medium plates for 4-6 days. Transverse cuts were made within a millimeter to the shoot apex and subsequently aligned on a $0.45 \mu \mathrm{m}$ nitrocellulose filter (Millipore) on top of two moist Whatman no. 1 
filter paper's layers. Sealed plates were positioned vertically for 7 days prior to transfer to hydroponic or normal $1 / 2$ MS media.

\section{Cloning procedures}

Cloning pSUC2::iCals3m involved multi-site gateway (Invitrogen). Entry vector p1R4pAtSUC2::XVE (Siligato et al., 2016), pDONR221-cals3m (Vatén et al., 2011) and p2R3a-3AT/nosT (Siligato et al., 2016) were recombined with the destination binary vector pCAM-hyg-R4R3 (Siligato et al., 2016). The CC-specific promoter pSUC2 was PCR-amplified from genomic DNA (primers in table S4) and recombined into the Gateway vector $p D O N R$ P4-P1R. For cloning $p A T M L 1:: S U L-I R$, the ATML1 promoter was PCR-amplified (primers ATML1promoter-F and ATML1promoter$\mathrm{R}$;Table S4) and inserted as an EcoRI-Xhol restriction fragment into pFGC5941. All final constructs were introduced into A. tumefaciens GV3101 for Arabidopsis transformation via floral dip (Clough and Bent, 1998).

\section{Immunoprecipitation}

Root tissue was ground in IP buffer (50mM Tris-HCL, pH7.5, 150mM NaCL, 10\% glycerol, $0.1 \%$ NP40) containing cOmplete protease inhibitor (Roche). After clearing lysates at $12,000 \times \mathrm{g}(10 \mathrm{~min})$, supernatants were transferred into fresh tubes and $10 \%$ vol used as input. Lysates were incubated with anti-AGO4 antibody (1:10000; Agrisera AS09 617) for 1-2hrs, upon which protein A/G magnetic beads (Pierce) were added before further incubatation for 1-2hrs. Beads were washed $3 x$ with IP buffer, proteins acetone-precipitated from the organic phase and RNA extracted as described below. 


\section{Protein extraction, gel blot analysis}

Proteins were resolved via SDS-PAGE, transferred to Immobilon-P PVDF membranes (Millipore) and subjected to the anti-AGO4 antibody (1:5000; Agrisera AS09 617) in blocking buffer (1x PBS, 0.1\% Tween-20, 5\% skim-milk powder). After washing, membranes were incubated for $1 \mathrm{hr}$ at RT with a horseradish peroxidaseconjugated anti-rabbit goat serum (1:10000; Abcam ab6721). After washing, detection was through the ECL Western Blotting Detection Kit (GE Healthcare) revealed via ChemiDocTouch from Bio-Rad. Coomassie blue-stained membranes provided loading controls.

\section{RNA isolation, cDNA synthesis and qRT-PCR}

Tri-Reagent was used for total/immunoprecipitated RNA extractions. Reverse transcription of DNasel-treated RNA was performed with the Maxima First Strand cDNA Synthesis Kit (Thermo). qRT-PCR was performed on LightCycler480II (Roche) using the KAPA-SYBR-FAST qPCR kit (Roche; Brosnan et al., 2019). Ct-values were determined by $2^{\text {nd }}$-derivative max on average of technical triplicates. Data are presented as average of three biological replicates calculated from $\Delta \mathrm{Ct}$ values obtained by comparing the gene-of-interest to a set of house-keeping genes. Normal distributions were confirmed by Shapiro-Wilk test $(\alpha=0.02)$. Equal variances were evaluated by F-test ( $95 \%$ confidence interval).

\section{RNA gel blot analysis}

Low-molecular-weight northern blots were conducted as described (Brosnan et al,. 2019). Total/immunoprecipitated RNA was resolved on $17.5 \%$ denaturing PAGE, electrotransferred to HyBond-Nylon NX (GE Healthcare) and EDC-crosslinked (Pall 
and Hamilton, 2008). Probes against individual miRNAs were generated by endlabelling with $\left[\mathrm{Y}^{-32} \mathrm{P}\right]-\mathrm{dATP}$ of complementary oligonucleotides . Probes against siRNA populations were PCR products labelled with the Prime-a-Gene kit (Promega). Over-night hybridization at $42^{\circ} \mathrm{C}$ was in PerfectHyb buffer (Sigma) followed by washing four times with $2 x$ SSC $0.1 \%$ SDS at $50^{\circ} \mathrm{C}$. Signals were revealed with a Typhoon FLA9500 scanner (GE Healthcare). For re-probing, membranes were stripped in boiling $0.1 \%$ SDS.

\section{Bisulfite sequencing}

As described (Pumplin et al., 2016), total genomic DNA was converted using the EZ DNA Methylation Kit (ZYMO Research) and amplified with primers listed in Table S4. The amplified DNA was mobilized into pGEM-Teasy, with at least 10 independent clones sequenced per treatment. Analysis was performed using Kismeth (Gruntman et al., 2008) (http://katahdin.mssm.edu/kismeth/revpage.pl). 95\% confidence intervals were calculated using Wilson score (Table S1).

\section{Small RNA sequencing}

RNA from SS-WT micro-grafting was processed into libraries using modified Illumina protocols by -and sequenced at- Fasteris (http://www.fasteris.com, Switzerland) using the HiSeq2000 sequencer. For WT and ir71 mutant libraries, RNA (10 $\mu$ l at 200ng/ $\mu$ l) was processed into libraries and sequenced on the HiSeq2500 sequencer at the Functional-Genomics-Center-Zurich (http://www.fgcz.ch/). 


\section{mRNA sequencing}

Total RNA from WT and ir71 epidermis was sequenced (random hexamer pairedend 2x125-bp-stranded RNA-sequencing) at Fasteris (http://www.fasteris.com, Switzerland) after ribosomal depletion.

\section{sRNA sequencing data processing and representation}

Small RNA data from grafting experiment are from GEO accession GSE112885 and have been processed as described in Devers et al. 2020. For WT and ir71 mutant libraries, reads were clipped using fastx_clipper from fastx-toolkit (http://hannonlab.cshl.edu/fastx toolkit/) looking for the adapter sequence's first 15nt(TGGAATTCTCGGGTG). 17-to-30-nt reads with identical sequences were grouped using the processReads function from the ncPRO-seq pipeline (Chen et al., 2012) and aligned against the $A$. thaliana genome (TAIR10) using Bowtie2 (Langmead and Salzberg, 2012) with options -k $100--$ score-min $\mathrm{L}, 0,0$.

In Fig. S6, simple genomic position comparison was applied to retrieve sRNA read counts and positions corresponding to $S S$ or $I R 71$, subsequently used to calculate normalized read-counts (reads per 1 millions mapped reads) for each nucleotide and to produce a graphical representation with $\mathrm{R}$. Color-coded reads-lengths are indicated in figures' legends.

\section{Quantification of TE-derived, graft-transmitted sRNAs}

To quantify TE-derived sRNA graft-transmission, we used data from Molnar et al., 2010 (GSM518438-40, GSM518443-45). Raw data were retrieved from GEO/SRA, adaptor sequences removed using fastx_clipper (-a TCGTATGCCGT) and processed 
as described above. TE being repeated sequences, in order to quantify the sRNA responsible for the signal obtained in Fig. 4A we first aligned the sequences corresponding to the probes to the TAIR10 genome using bowtie2. Then, sRNA reads overlapping the inverse strand of the obtained region, enlarged by $5 \mathrm{bp}$ on both sides, were selected using intersectBed from bedtools (v2.25.0; Quinlan and Hall, 2010) with options $-S-F \quad 0.9$. For each region, read counts were normalized (reads per million mapped reads) and the average between replicates was represented as boxplot with $\mathrm{R}$. Mean values for each type of TE sequences were indicated in red.

\section{mRNA sequencing data processing}

Reads were trimmed using Trimmomatic (v0.36; (Bolger et al., 2014)) with parameters ILLUMINACLIP:TruSeq2-PE.fa:2:30:10 SLIDINGWINDOW:4:15 MINLEN :50 and mapped to TAIR10 Ensembl-genome-and-genes-annotation (http://ccb.jhu.edu/software/tophat/igenomes.shtml) using TopHat (v2.0.11; (Trapnell et al., 2009) and Bowtie2 (v2.2.1.0; Langmead and Salzberg, 2012). Counts for annotated genes (Ensembl TAIR10) were generated using the featurecounts function from Subread (Liao et al., 2014) with options - S rf -p - s 1 - C - B - O. Differential analysis and MAplot in Fig. 5D were done with DEseq2 (v1.12.4; (Love et al., 2014) taking into account the two replicates. Genes with adjusted p-values $<0.01$ were considered differentially expressed.

\section{Gene Ontology enrichment}

Up- and down-regulated genes were subjected to agriGO gene-ontology-enrichment analysis (Tian et al., 2017) using the TAIR10 Arabidopsis reference genome and 
default parameters. Enriched GO terms were then transferred to REVIGO (Supek et al., 2011) with parameters: Similarity Medium ; database : Arabidopsis thaliana ; semantic similarity measure : Resnik (normalized). Only terms with a frequency $<1$ were considered. Histograms in Fig. 5E-F represent the proportion of genes in the query list and background for the top 10 ontology terms following ranking based on $\log _{10}$ ( $p$-value).

\section{Leaf epidermis peeling (Meselect)}

As described (Svozil et al., 2016), the lower epidermis was removed by peeling after placing leaves between two sticky tapes. Residual mesophyll cells were removed in protoplasting solution (1\% cellulase Onozuka RS (Yakult), 0.4M mannitol, 10mM $\mathrm{CaCl} 2,20 \mathrm{mM} \mathrm{KCl}, 0.1 \% \mathrm{BSA}$ and 20mM MES, $\mathrm{pH} 5.7)$. The epidermal side was incubated for 15min and the vasculature for 20-30min; separated tissues were washed twice with ice-cold wash buffer $(154 \mathrm{mM} \mathrm{NaCl}, 125 \mathrm{mM} \mathrm{CaCl} 2,5 \mathrm{mM} \mathrm{KCl}$, $5 \mathrm{mM}$ glucose and $2 \mathrm{mM}$ MES, $\mathrm{pH} 5.7)$. The vasculature was removed with forceps and frozen in liquid nitrogen. RNA was extracted as described above.

\section{Callose staining}

Plants were fixed in acetic acid/ethanol 1:3 (v/v) for at least $2 \mathrm{hrs}$ and washed three times for $20 \mathrm{~min}$ in $150 \mathrm{mM} \mathrm{K}_{2} \mathrm{HPO}_{4}$. Aniline blue $0.01 \%(\mathrm{w} / \mathrm{v})$ in $150 \mathrm{mM} \mathrm{K}_{2} \mathrm{HPO}_{4}$ was used to stain callose depositions overnight at RT protected from light. Stained callose structures were imaged using an epifluorescence microscope with a DAPI filter.

\section{Data Availability}


Data have been deposited into the Gene Expression Omnibus (GEO) under the accession number GSE112929 for the RNA-sequencing data from WT and ir71 epidermis and under the accession number GSE112861 for the sRNA-sequencing from WT and ir71 shoot apex. 


\section{References}

Bolger, A.M., Lohse, M., and Usadel, B. (2014). Trimmomatic: a flexible trimmer for Illumina sequence data. Bioinformatics 30, 2114-2120.

Brosnan, C.A., Mitter, N., Christie, M., Smith, N.A., Waterhouse, P.M., and Carroll, B.J. (2007). Nuclear gene silencing directs reception of long-distance mRNA silencing in Arabidopsis. Proc. Natl. Acad. Sci. U.S.A. 104, 14741-14746.

Brosnan, C.A., Sarazin, A., Lim, P., Bologna, N.G., Hirsch-Hoffmann, M., and Voinnet, O. (2019). Genome-scale, single-cell-type resolution of microRNA activities within a whole plant organ. EMBO J. 38, e100754.

Chen, C.-J., Servant, N., Toedling, J., Sarazin, A., Marchais, A., Duvernois-Berthet, E., Cognat, V., Colot, V., Voinnet, O., Heard, E., et al. (2012). ncPRO-seq: a tool for annotation and profiling of ncRNAs in sRNA-seq data. Bioinformatics 28, 31473149.

Clough, S.J., and Bent, A.F. (1998). Floral dip: a simplified method for Agrobacterium-mediated transformation of Arabidopsis thaliana. Plant J. 16, 735743.

Deleris, A., Gallego-Bartolome, J., Bao, J., Kasschau, K.D., Carrington, J.C., and Voinnet, O. (2006). Hierarchical action and inhibition of plant Dicer-like proteins in antiviral defense. Science 313, 68-71.

Devers, E.A., Brosnan, C.A., Sarazin, A., Albertini, D., Amsler, A.C., Brioudes, F., Jullien, P.E., Lim, P., Schott, G., and Voinnet, O. (2020). Movement and differential consumption of short interfering RNA duplexes underlie mobile RNA interference. Nat. Plants.

Gibeaut, D.M., Hulett, J., Cramer, G.R., and Seemann, J.R. (1997). Maximal biomass of Arabidopsis thaliana using a simple, low-maintenance hydroponic method and favorable environmental conditions. Plant Physiol. 115, 317-319.

Gruntman, E., Qi, Y., Slotkin, R.K., Roeder, T., Martienssen, R.A., and Sachidanandam, R. (2008). Kismeth: analyzer of plant methylation states through bisulfite sequencing. BMC Bioinformatics 9, 371.

Himber, C., Dunoyer, P., Moissiard, G., Ritzenthaler, C., and Voinnet, O. (2003). Transitivity-dependent and -independent cell-to-cell movement of RNA silencing. EMBO J. 22, 4523-4533.

Langmead, B., and Salzberg, S.L. (2012). Fast gapped-read alignment with Bowtie 2. Nat. Methods 9, 357-359.

Love, M.I., Huber, W., and Anders, S. (2014). Moderated estimation of fold change and dispersion for RNA-seq data with DESeq2. Genome Biol 15, 550. 
Molnar, A., Melnyk, C.W., Bassett, A., Hardcastle, T.J., Dunn, R., and Baulcombe, D.C. (2010). Small silencing RNAs in plants are mobile and direct epigenetic modification in recipient cells. Science $328,872-875$.

Pall, G.S., and Hamilton, A.J. (2008). Improved northern blot method for enhanced detection of small RNA. Nat Protoc 3, 1077-1084.

Pumplin, N., Sarazin, A., Jullien, P.E., Bologna, N.G., Oberlin, S., and Voinnet, O. (2016). DNA Methylation Influences the Expression of DICER-LIKE4 Isoforms, Which Encode Proteins of Alternative Localization and Function. Plant Cell 28, 2786-2804.

Siligato, R., Wang, X., Yadav, S.R., Lehesranta, S., Ma, G., Ursache, R., Sevilem, I., Zhang, J., Gorte, M., Prasad, K., et al. (2016). MultiSite Gateway-Compatible Cell Type-Specific Gene-Inducible System for Plants. Plant Physiol. 170, 627-641.

Smith, L.M., Pontes, O., Searle, I., Yelina, N., Yousafzai, F.K., Herr, A.J., Pikaard, C.S., and Baulcombe, D.C. (2007). An SNF2 protein associated with nuclear RNA silencing and the spread of a silencing signal between cells in Arabidopsis. Plant Cell 19, 1507-1521.

Stadler, R., Wright, K.M., Lauterbach, C., Amon, G., Gahrtz, M., Feuerstein, A., Oparka, K.J., and Sauer, N. (2005). Expression of GFP-fusions in Arabidopsis companion cells reveals non-specific protein trafficking into sieve elements and identifies a novel post-phloem domain in roots. Plant J. 41, 319-331.

Supek, F., Bošnjak, M., Škunca, N., and Šmuc, T. (2011). REVIGO summarizes and visualizes long lists of gene ontology terms. PLoS One 6, e21800.

Svozil, J., Gruissem, W., and Baerenfaller, K. (2016). Meselect - A Rapid and Effective Method for the Separation of the Main Leaf Tissue Types. Front Plant Sci 7, 1701.

Tian, T., Liu, Y., Yan, H., You, Q., Yi, X., Du, Z., Xu, W., and Su, Z. (2017). agriGO v2.0: a GO analysis toolkit for the agricultural community, 2017 update. Nucleic Acids Res 45, W122-W129.

Tocquin, P., Corbesier, L., Havelange, A., Pieltain, A., Kurtem, E., Bernier, G., and Périlleux, C. (2003). A novel high efficiency, low maintenance, hydroponic system for synchronous growth and flowering of Arabidopsis thaliana. BMC Plant Biol. 3, 2.

Trapnell, C., Pachter, L., and Salzberg, S.L. (2009). TopHat: discovering splice junctions with RNA-Seq. Bioinformatics 25, 1105-1111.

Turnbull, C.G.N., Booker, J.P., and Leyser, H.M.O. (2002). Micrografting techniques for testing long-distance signalling in Arabidopsis. Plant J. 32, 255-262.

Vatén, A., Dettmer, J., Wu, S., Stierhof, Y.-D., Miyashima, S., Yadav, S.R., Roberts, C.J., Campilho, A., Bulone, V., Lichtenberger, R., et al. (2011). Callose biosynthesis regulates symplastic trafficking during root development. Dev. Cell 21, 1144-1155. 
bioRxiv preprint doi: https://doi.org/10.1101/2021.10.06.463290; this version posted October 6, 2021. The copyright holder for this preprint (which was not certified by peer review) is the author/funder. All rights reserved. No reuse allowed without permission. 


\section{Supplementary Figure Legends}

Figure S1. Photobleaching phenotype in source versus sink leaves of SS transgenic Arabidopsis. 4 weeks old soil grown Arabidopsis plant with typical photobleaching phenotype due to RNAi triggered by expression of pSUC2::SUL-IR (SS). The letters indicate the position of the extracted leaves on the intact plant. From oldest to youngest leaf $A$ corresponds to $L 5$ and $B$ to $L 11$ and were designated as source leaf (SoL) and sink leaf (SiL), respectively. Roman numerals and black arrows indicate the leaf veins of class I (mid-rib), II (veins of second order, originating from the mid-rib) and III (veins of third order, originating from class II veins). Scale bars represent $5 \mathrm{~mm}$. For better comparison, the background in those pictures was masked but original photos used in this figure can be found as supplementary data files.

Figure S2. Phenotypic quantification and distribution of callose depositions at vascular bundles in iSC expressing lines. (a) Phenotypic scoring of the $S S$ bleaching phenotype of each leaf stage in populations of iSC (left) and $S S$ (right) plants. Yellow bars represent the percentage of the population showing vein centered bleaching of chlorophyll, green bars represent the percentage of the population showing no signs of vein centered bleaching and grey bars represent the percentage of the population having the respective leaf missing at the time of screening. Error bars represent SE, $\mathrm{n}=4$ (iSC) and 7 (SS) populations of 6-12 plants. (b) Epifluorescence images of an aniline blue stained leaf series of iSC plant lines grown on either control (left) or inducing (right) media. Concentrations correspond to 17-ß-estradiol present in the media. Scale bars represent $200 \mu \mathrm{m}$. Co 
= cotyledon; $\mathrm{L}=$ leaf. (c) qRT-PCR analysis of endogenous SUC2 and DCL4 mRNA levels in mock- or ß-estradiol-induced seedlings. Error bars represent SE, $\mathrm{n}=6-10$. Asterisks indicate statistically significant differences $(p \leq 0.05$, two-tailed Student's ttest with equal variance [F-test, $p>0.05])$.

Figure S3. Representative images equivalent aged flowering WT (left), SS (middle) and amiRSUL (right) plants. Insets show lack of photobleaching in stem tissue of each respective genotype.

Figure S4. Comparison of Argonaute transcript levels in various tissues and cell types of Arabidopsis. Analysis of publicly available transcriptome data to compare transcript levels of AGO1, 2, 4 and 6 in (a) different plant tissues using the EMBL-EBI Gene expression atlas (https://www.ebi.ac.uk/gxa/home; release 37) (source data from PMID: 23136377) or (b) in different leaf cell-types manually extracted from data published in Berkowitz et al., 2021.

Figure S5. Analysis of sequenced graft-transmissible sRNA populations. Boxplot representation of sRNA abundance (read per millions mapped reads) corresponding to 3 classes of repeat sequences (Rep2, 1003 and SimpleHat). Sequencing libraries correspond to graft-transmissible sRNA from Molnal et al., 2021. WT/WT refers to $\mathrm{Col} / \mathrm{Col}$ and dcl234 refers to Col-dcl2,3,4 of the original nomenclature.

Figure S6. siRNA profiles of mobile SS populations and IR71 knockout loci. (a) Profile and histograms of SS-derived siRNA reads from grafted WT scion (Top) on 
top of a SS rootstock (Bottom). (b) Upper panels show the profile and size distribution (right) of IR71-derived siRNAs in WT plants. Middle panel shows siRNA profile of IR71-derived siRNAs in T-DNA line (ir71 -/-). The lower panel shows the siRNA profile of IR71-derived siRNAs in T-DNA line (ir71 -/-) with adjusted scale demonstrating background levels of siRNA. Top and middle panels are also shown in Figure 4. RPM - Reads per Million, color code: Blue - 20-21-nt, Green - 22-23-nt and Red - 24-25-nt. 
bioRxiv preprint doi: https://doi.org/10.1101/2021.10.06.463290; this version posted October 6, 2021. The copyright holder for this preprint (a) which was not certified by peer review) is the author/funder. All riahts reserved. No reuse allowed without permission.

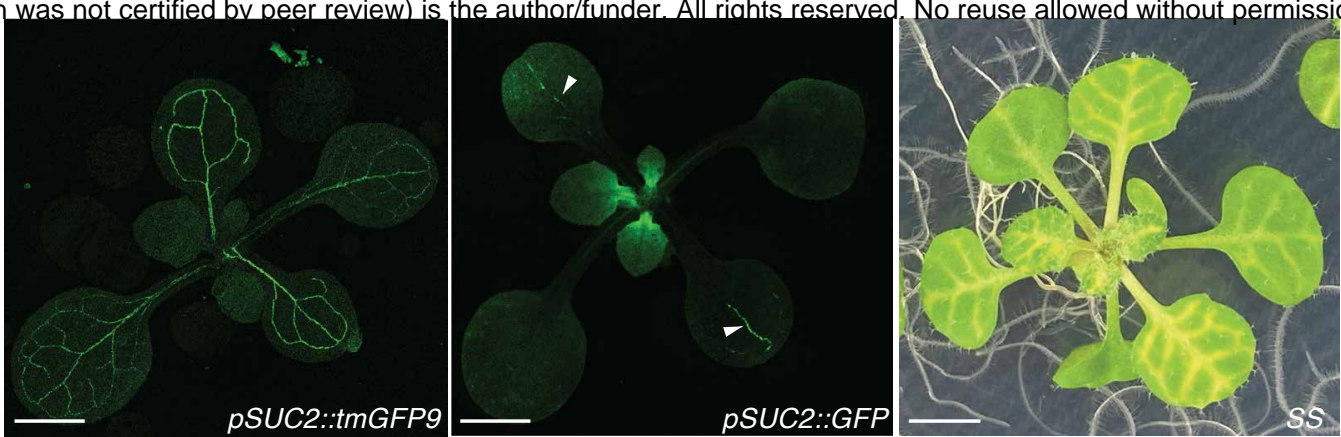

(b)
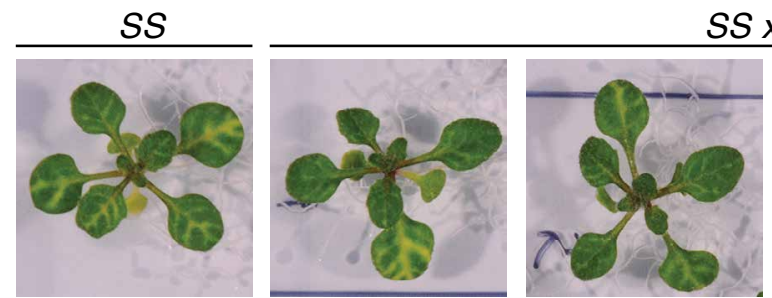

SS $\times$ iSC
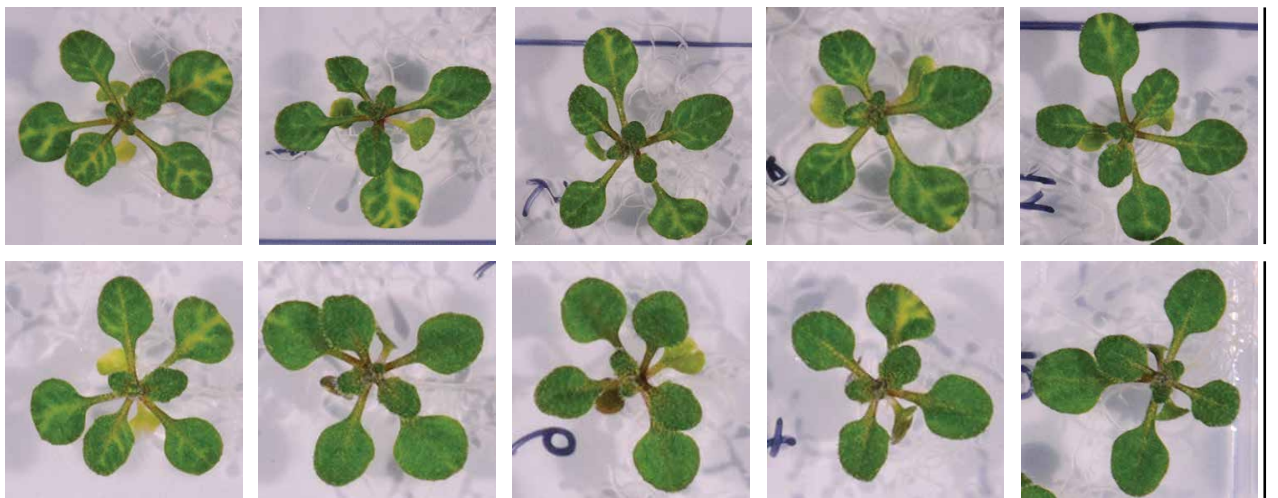

mock

(c)
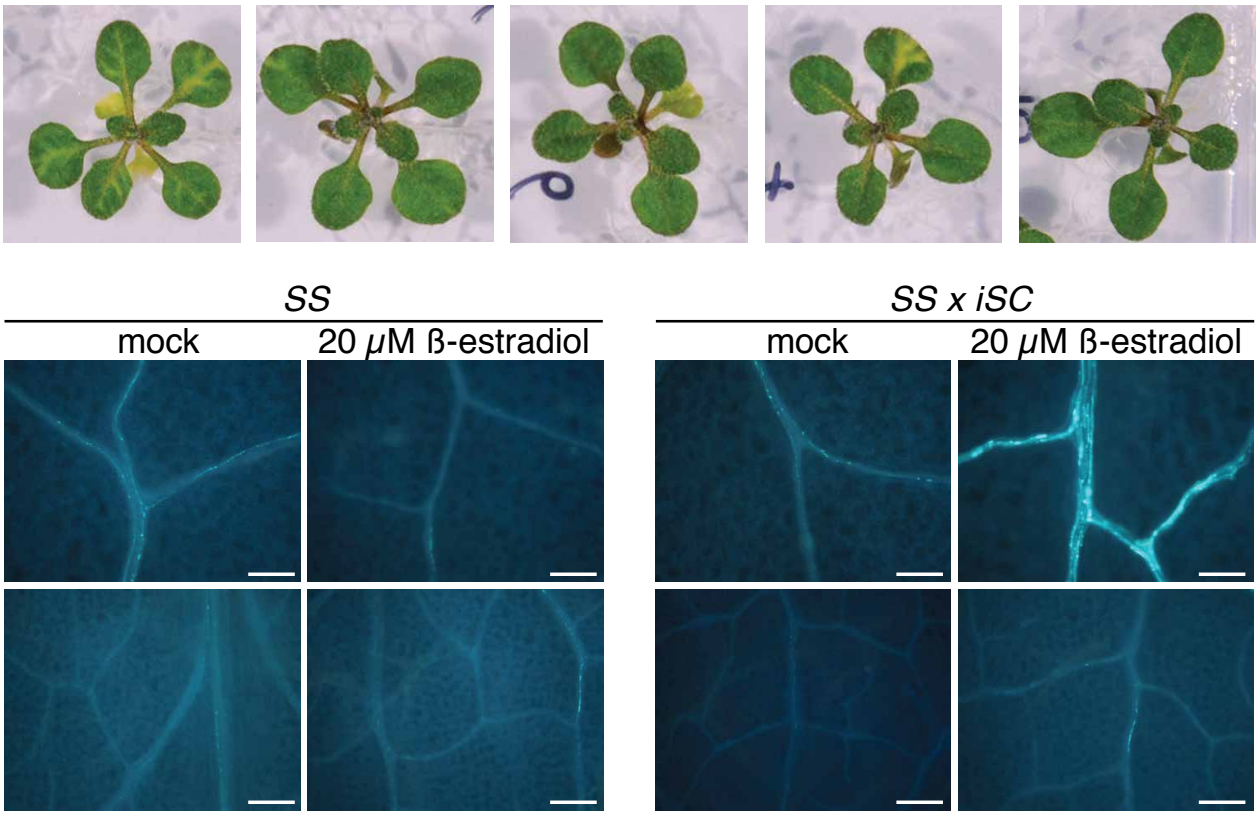

B-estradiol

(d)
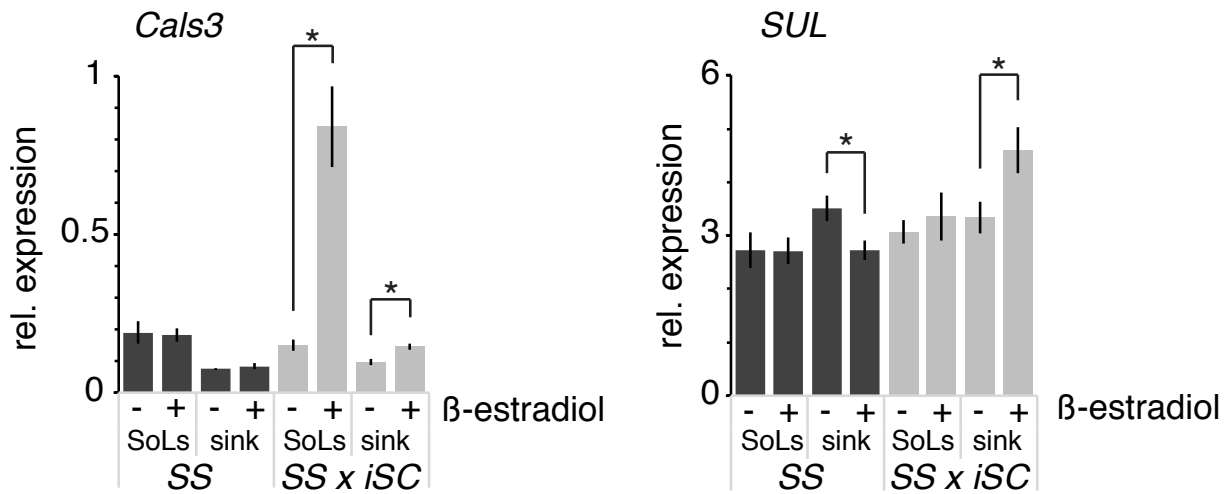

(e)

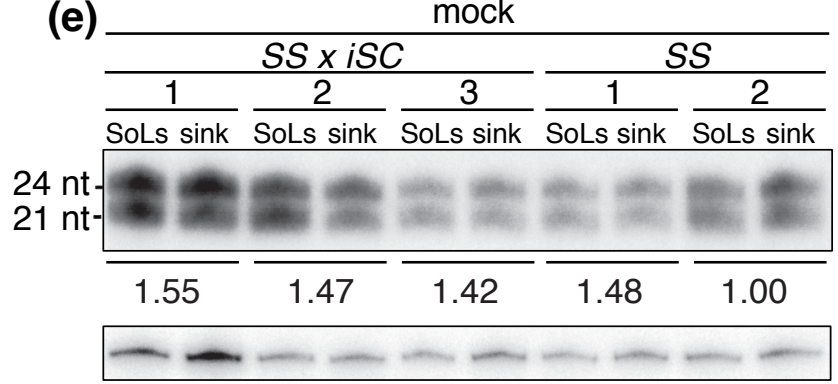

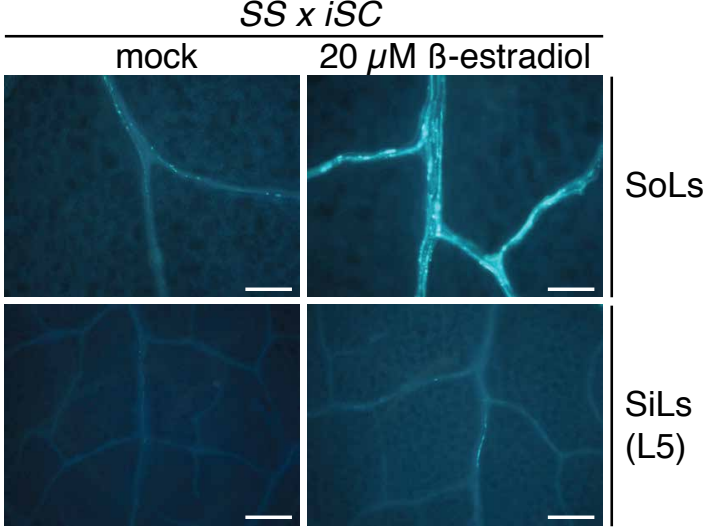

L5)

\section{Figure 1}


(a)

(b)

bioRxiy preprint doi: https://doi.org/10.1101/2b21.10,88.46

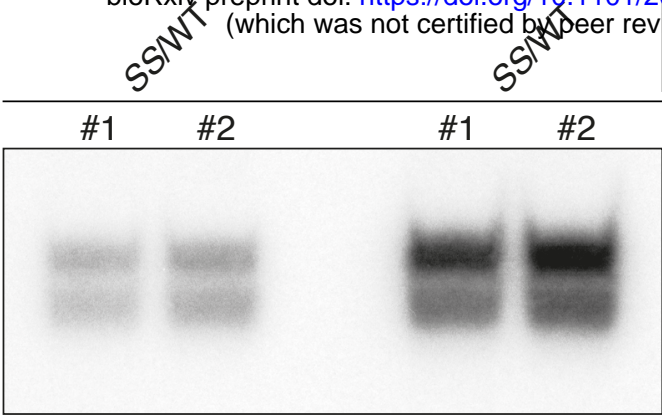

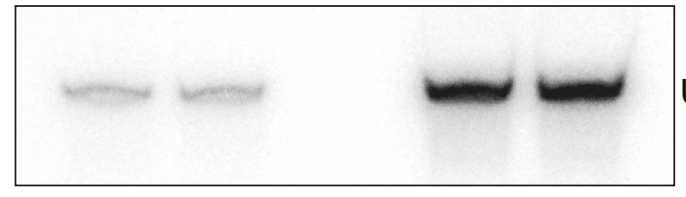

U6

Shoot

Root

SUL
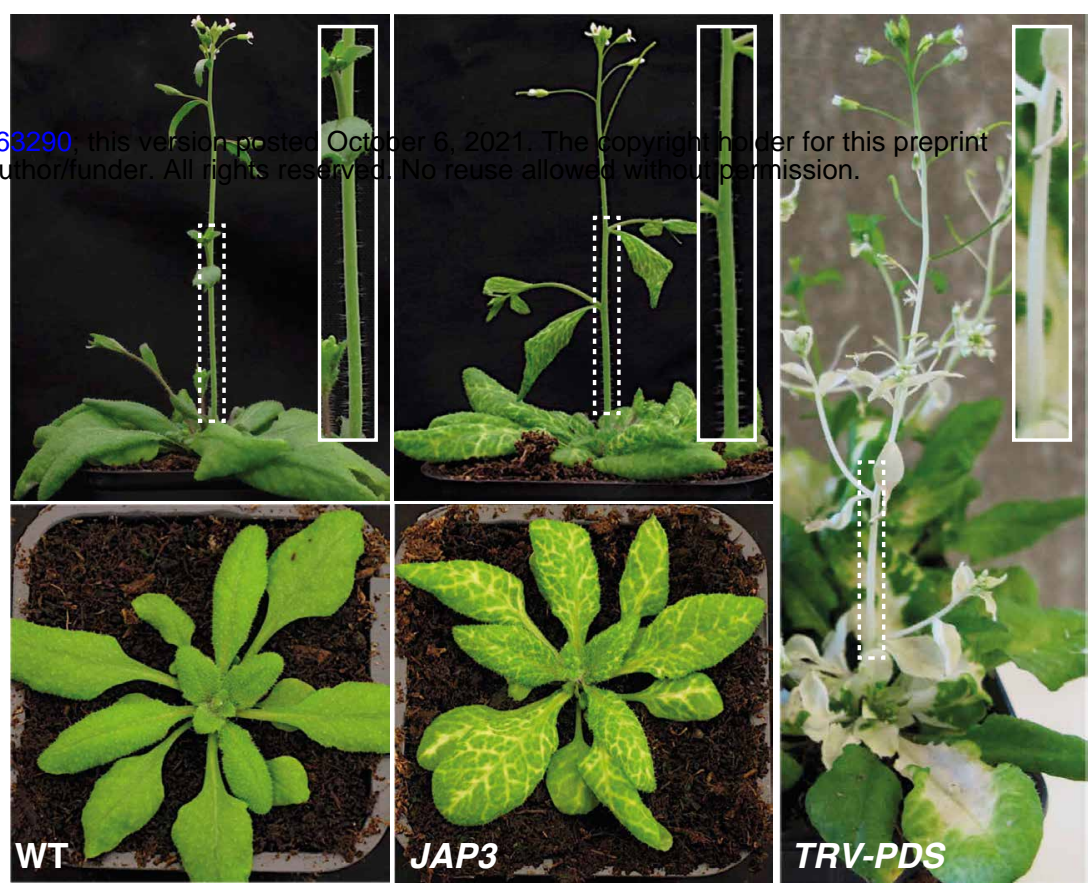

(c)
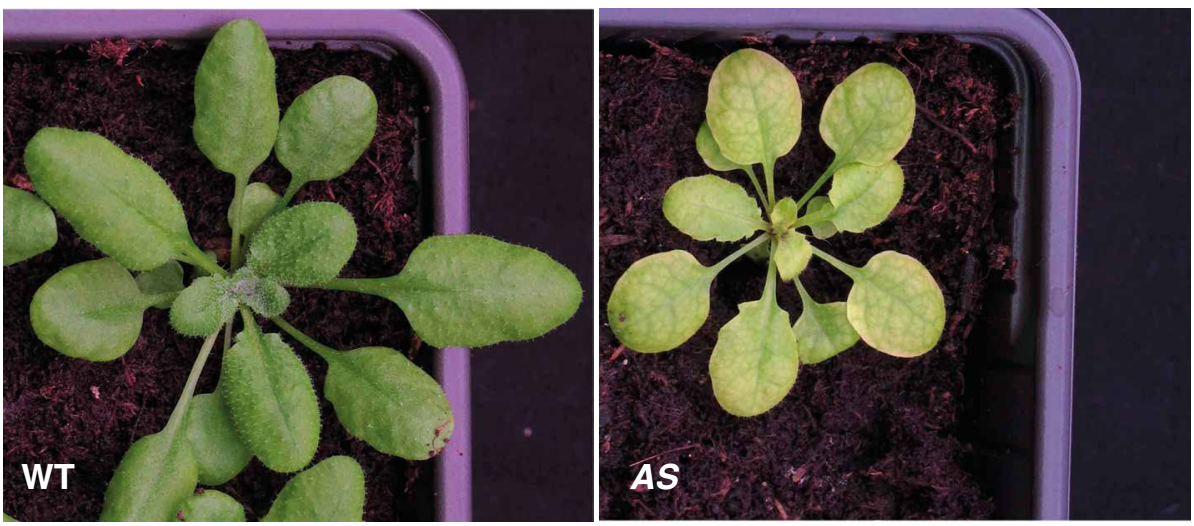

(d)

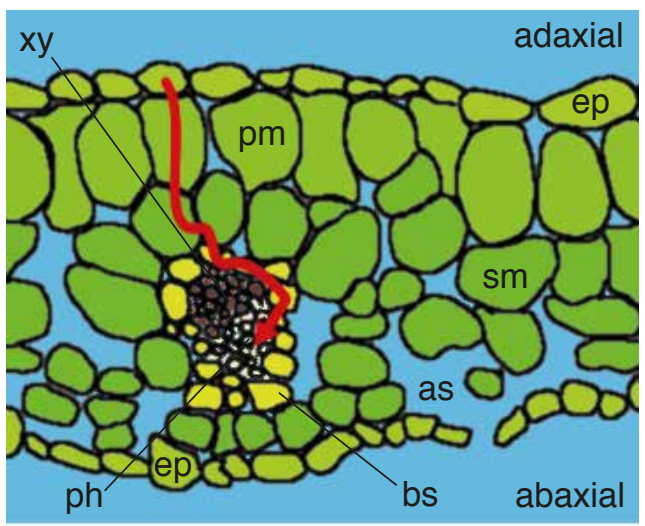

(e)
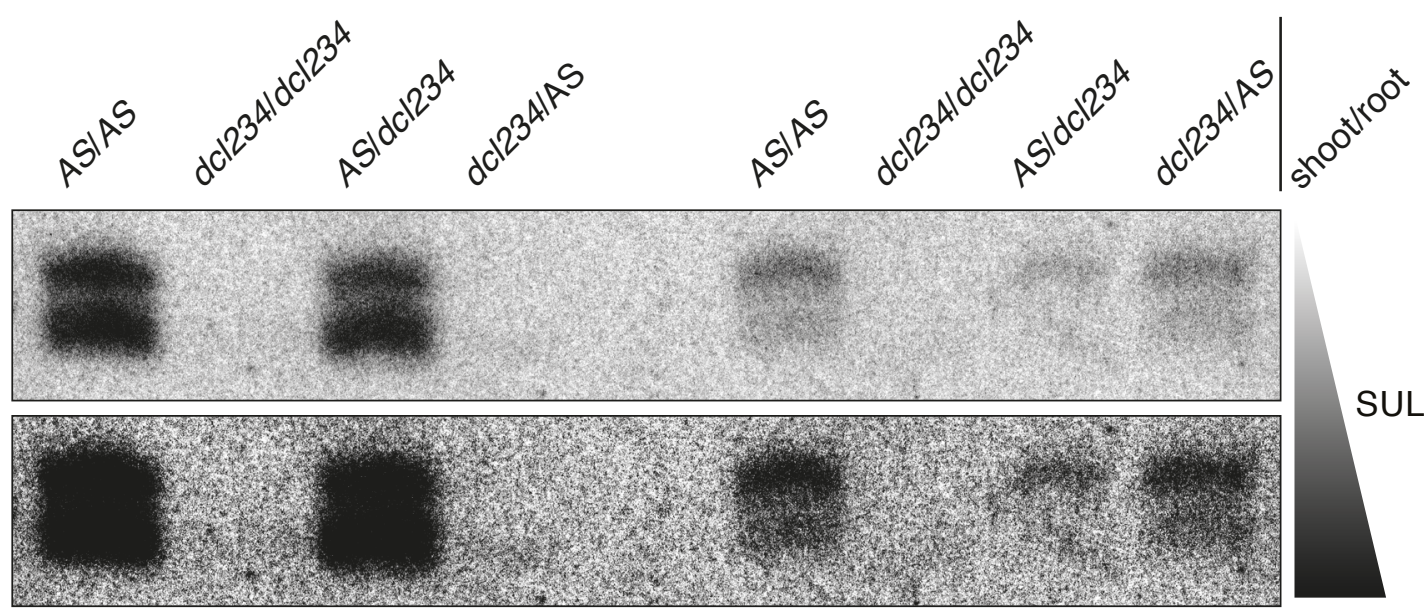

SUL

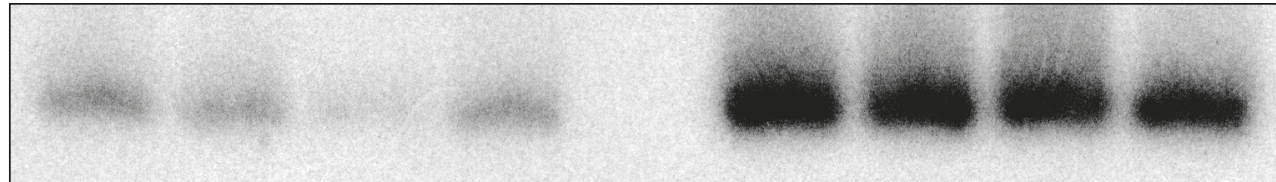

miR160

$\longrightarrow-\cdots$

$\square-0$

U6

Shoot

Root

Figure 2 
(a)

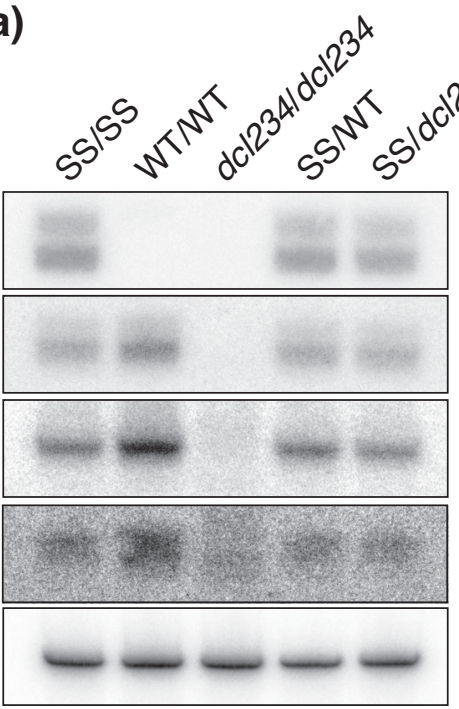

Shoot
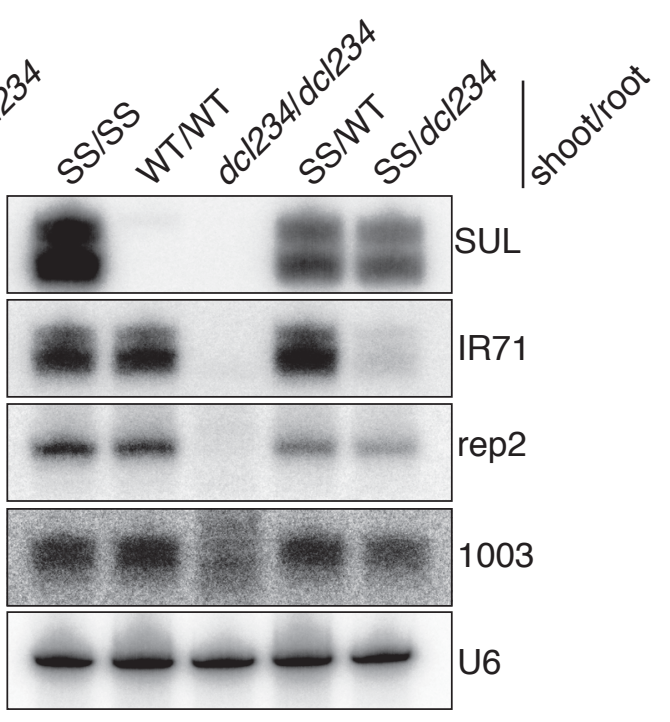

Root (b)

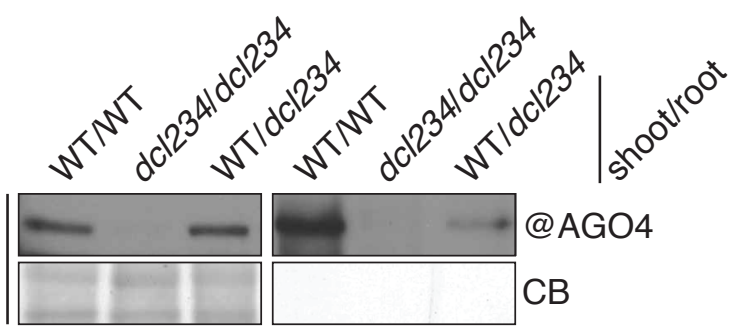

RNA

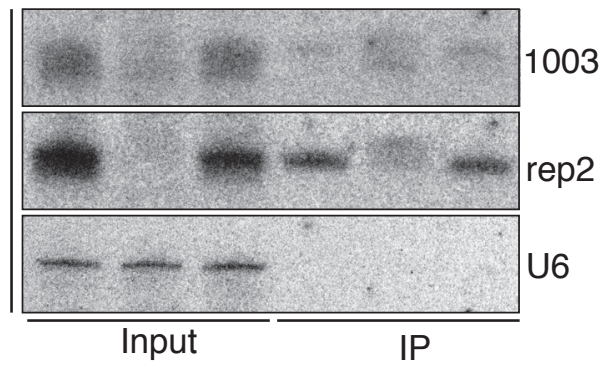

(c)

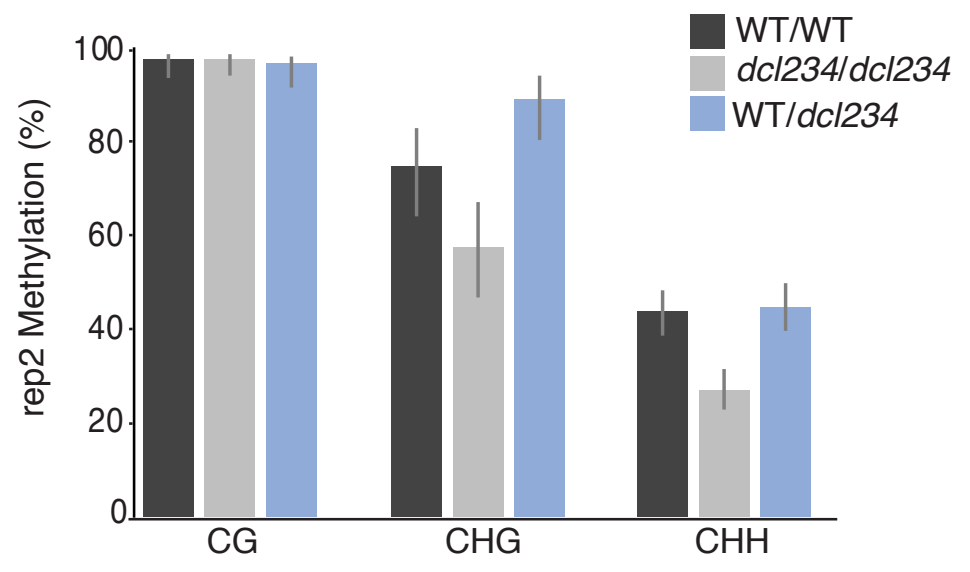

Figure 3 
(a)

(b)

WT

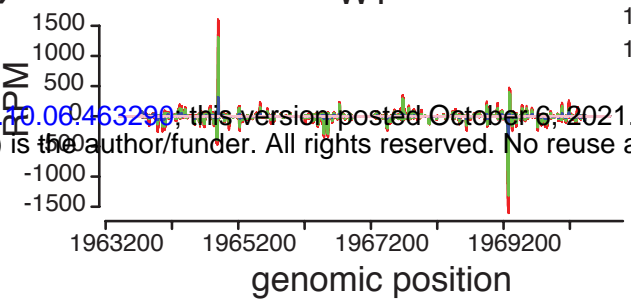

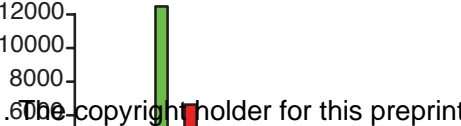

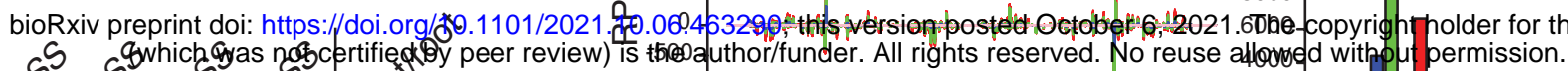

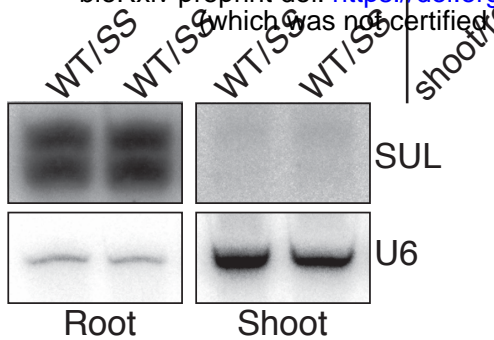

2000 genomic position
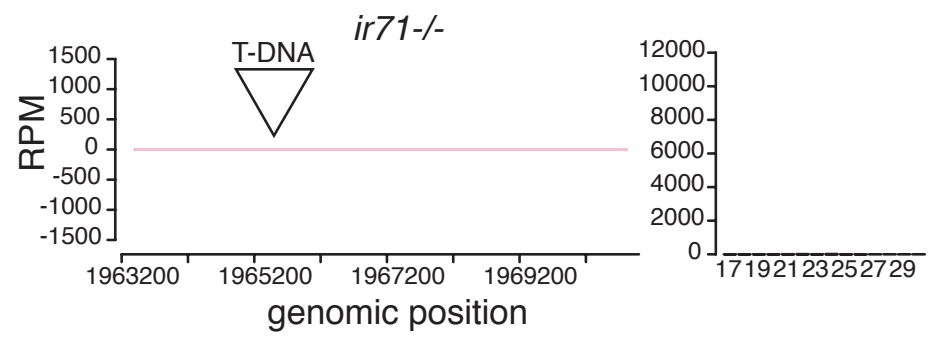
genomic position

(c)

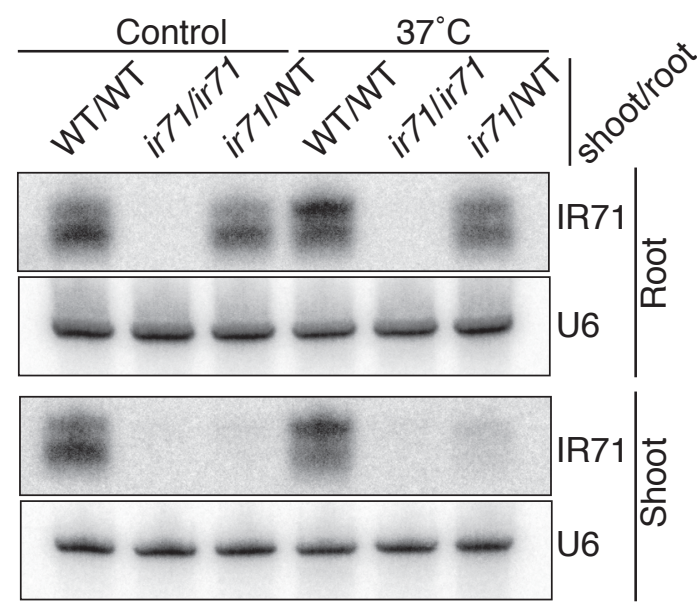

(d)

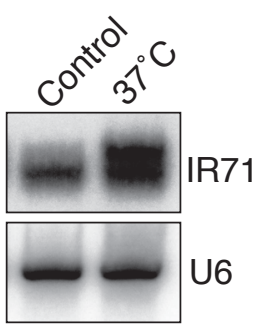

(e)

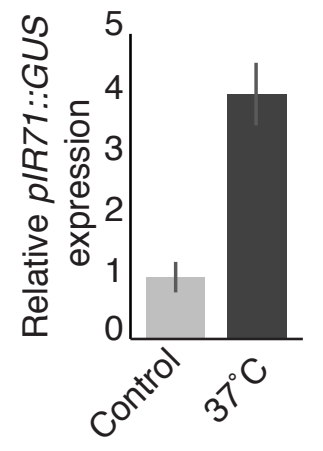

Figure 4 
bioRxiv preprint doi: https://doi.org/10.1101/2021.10.06.463290; this version posted October 6, 2021. The copyright holder for this preprint

(a)

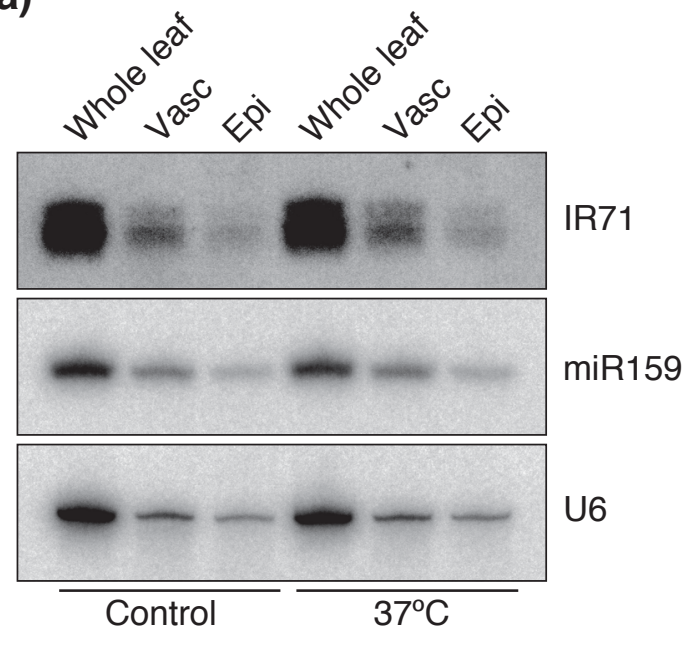

(b) (c)

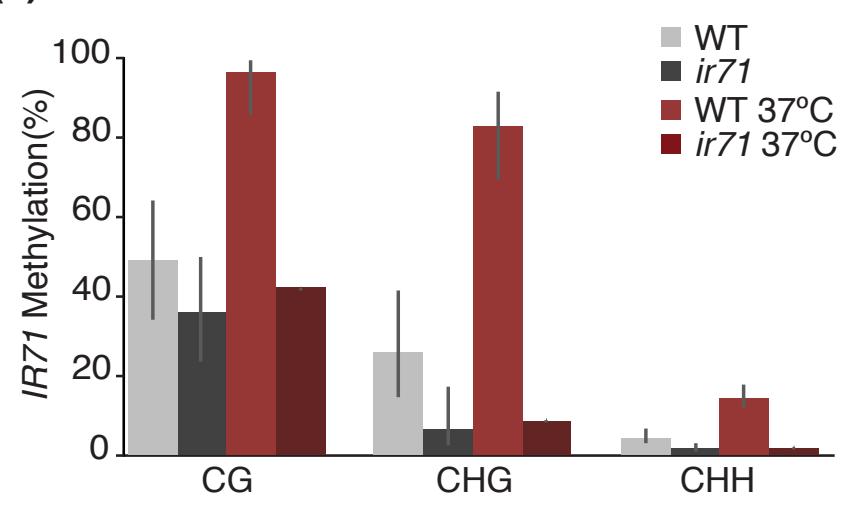

(d)

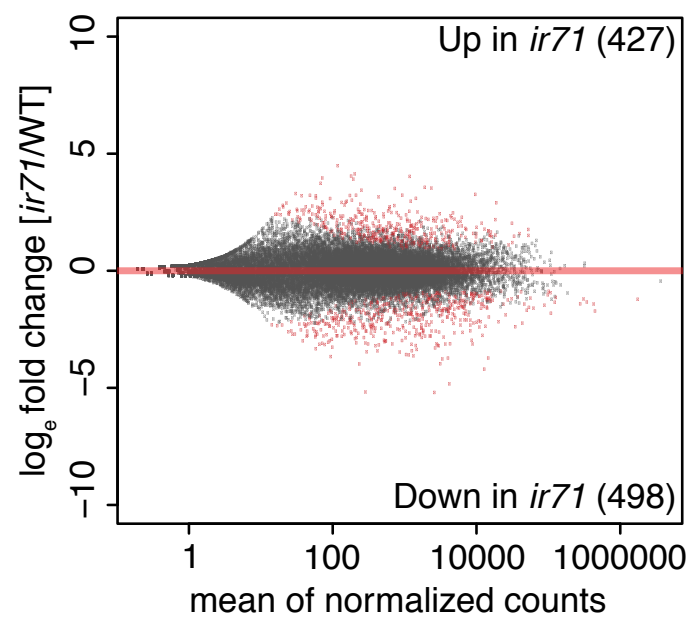

(e)

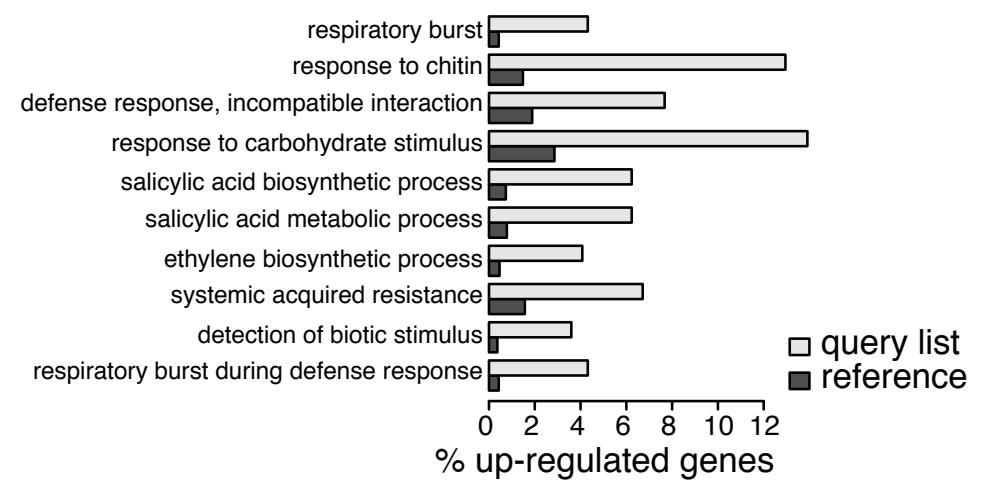

(f)

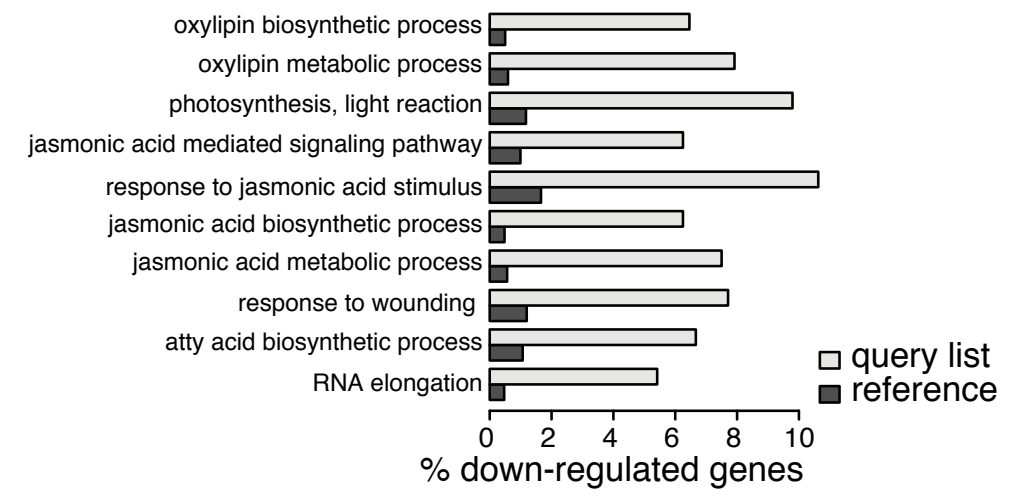

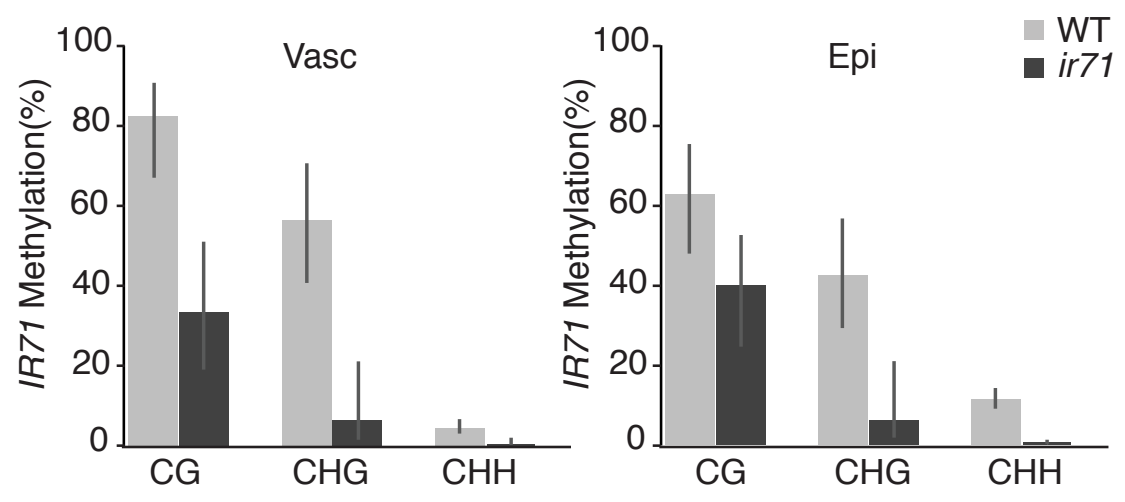

